July 10, 2018

\title{
Inclusive Nucleon Emission Induced by Quasi-Elastic Neutrino-Nucleus Interactions.
}

\author{
J. Nieves, ${ }^{1}$ M. Valverde, ${ }^{1}$ and M.J. Vicente Vacas $^{2}$ \\ ${ }^{1}$ Departamento de Fúsica Moderna, \\ Universidad de Granada, E-18071 Granada, Spain \\ ${ }^{2}$ Departamento de Física Teórica and IFIC, Centro Mixto Universidad de Valencia-CSIC \\ Institutos de Investigación de Paterna, Aptdo. 22085, E-46017 Valencia, Spain
}

\begin{abstract}
We study the quasi-elastic contribution to the inclusive $\left(\nu_{l}, \nu_{l} N\right),\left(\nu_{l}, l^{-} N\right),\left(\bar{\nu}_{l}, \bar{\nu}_{l} N\right)$ and $\left(\bar{\nu}_{l}, l^{+} N\right)$ reactions in nuclei using a Monte Carlo simulation method to account for the rescattering of the outgoing nucleon. As input, we take the reaction probability from the microscopical many body framework developed in Phys. Rev. C70 (2004) 055503 for charged-current induced reactions, while for neutral currents we use results from a natural extension of the model described in that reference. The nucleon emission process studied here is a clear signal for neutral-current neutrino driven reactions, that can be used in the analysis of future neutrino experiments.
\end{abstract}

PACS numbers: 25.30.Pt,13.15.+g, 24.10.Cn,21.60.Jz

\section{INTRODUCTION}

Neutrino physics is at the forefront of current theoretical and experimental research in astro, nuclear, and particle physics. Indeed, neutrino interactions offer unique opportunities for exploring fundamental questions in these domains of the physics. One of these questions is the neutrino-oscillation phenomenon, for which there have been conclusive positive signals in the last years [1]. Neutrino-oscillation experiments are presently evolving from the discovery to the precision phase. This new generation of experiments faces a major difficulty: the elusive nature of the neutrinos. The presence of neutrinos, being chargeless particles, can only be inferred by detecting the secondary particles they create when colliding and interacting with matter. Nuclei are often used as neutrino detectors, thus a trustable interpretation of neutrino data heavily relies on detailed and quantitative knowledge of the features of the neutrinonucleus interaction [2]. For instance, in the case of neutrino processes driven by the electroweak Neutral Current (NC), the energy spectrum and angular distribution of the ejected nucleons are the unique observables. There is a general consensus among the theorists that a simple Fermi gas model, widely used in the analysis of neutrino oscillation experiments, fails to provide a satisfactory description of the measured cross sections, and inclusion of further nuclear effects is needed [3].

Simultaneously, in recent years there have also been some initiatives aiming at understanding the quark and gluon substructure of the nucleon. The flavor dependence of the nucleon's spin is a significant fundamental question that is not yet fully understood. Experiments $[4,5]$ measuring the spin content of the nucleon have reported conflicting results on the amount of nucleon spin carried by strange quarks [6]. Recently, the FINeSSE collaboration at Fermilab has suggested $[7,8]$ that Quasi-Elastic (QE) neutrino-nucleus scattering, observed using a novel detection technique, provides a theoretically clean measure of this quantity. In this context, it is also necessary to control nuclear effects.

At intermediate energies, above the nuclear giant resonance and below the $\Delta(1232)$ regions ${ }^{1}$, neutrino-nucleus interactions have been studied within several approaches. Several different Fermi gas, Random Phase Approximation (RPA), shell model and superscaling based calculations have been developed during the last 15 years [19]-[38]. Some of these approaches have been also employed to compute neutrino or antineutrino induced single-nucleon emission cross sections. Most of the calculations use the plane wave and distorted wave impulse approximations (PWIA and DWIA, respectively), including or not relativistic effects. The PWIA calculations neglect all types of interactions between the ejected nucleon and the residual nuclear system, and therefore such a framework constitutes a poor approximation to evaluate nucleon emission cross sections. However, the PWIA has been often used to compute the ratio of proton $(\nu, p)$ to neutron $(\nu, n)$ yields, which at low neutrino energies and for light nuclei might be rather insensitive to rescattering effects.

\footnotetext{
1 There exists an abundant literature studying these two regions. See for instance a recent paper [9] on the excitation of nuclear giant resonances in neutrino scattering off nuclei or the older works of Refs. [10]-[15] also studying QE neutrino-nucleus scattering at low energies. On the $\Delta$-excitation in neutrino reactions, there are works, among others, by Alvarez-Ruso and collaborators [16], S.K. Singh and collaborators [17] and Lalakulich y Paschos [18].
} 
Within the DWIA the ejected nucleon is described as a solution to the Dirac or Schrödinger equation with an optical potential obtained by fitting elastic proton-nucleus scattering data. The imaginary part accounts for the absorption into unobserved channels ${ }^{2}$. This scheme, first developed in $\left(e, e^{\prime} p\right)$ studies where the final nucleus is left in the ground or in a particular excited state, is incorrect to study nucleon emission processes where the state of the final nucleus is totally unobserved, and thus all final nuclear configurations, either in the discrete or on the continuum, contribute. The distortion of the nucleon wave function by a complex optical potential removes all events where the nucleons collide with other nucleons. Thus, in DWIA calculations, the nucleons that interact are lost when in the physical process they simply come off the nucleus with a different energy, angle, and maybe charge, and they should definitely be taken into account. A clear example which illustrates the deficiencies of the DWIA models is the neutron emission process: $\left(\nu_{l}, l^{-} n\right)$. Within the impulse approximation neutrinos only interact via Charged Current (CC) interactions with neutrons and would emit protons, and therefore the DWIA will predict zero cross sections for CC one neutron knock-out reactions. However, the primary protons interact strongly with the medium and collide with other nucleons which are also ejected. As a consequence there is a reduction of the flux of high energy protons but a large number of secondary nucleons, many of them neutrons, of lower energies appear.

The distortion by a real potential does not eliminate the events where there are nucleon collisions. But, it does not account either for the changes of energy, direction and charge of the nucleons induced by these collisions. Besides, it cannot account either for more than one nucleon knock-out events.

In this work, we study the $\mathrm{QE}$ contribution to the inclusive $\left(\nu_{l}, \nu_{l} N\right),\left(\nu_{l}, l^{-} N\right),\left(\bar{\nu}_{l}, \bar{\nu}_{l} N\right)$ and $\left(\bar{\nu}_{l}, l^{+} N\right)$ reactions in nuclei. We use a Monte Carlo (MC) simulation method to account for the rescattering of the outgoing nucleon. A reliable description of the gauge bosons $\left(W^{ \pm}\right.$and $\left.Z^{0}\right)$ absorption in the nucleus is the first essential ingredient.

For CC driven processes, we use the many body framework developed in Ref. [34]. Starting from a Local Fermi Gas (LFG) picture of the nucleus, which automatically accounts for Pauli blocking, several nuclear effects are taken into account in the scheme of Ref. [34]: i) a correct energy balance, using the experimental $Q$-values, is enforced, ii) Coulomb distortion of the charged leptons is implemented by using the so called "modified effective momentum approximation" [40], iii) medium polarization (RPA), including $\Delta$-hole degrees of freedom and explicit pion and rho exchanges in the vector-isovector channel of the effective nucleon-nucleon force, and Short Range Correlation (SRC) effects are computed, and finally iv) the nucleon propagators are dressed in the nuclear medium, which amounts to work with a LFG of interacting nucleons and it also accounts for reaction mechanisms where the gauge boson, $W^{+}$ or $W^{-}$, is absorbed by two nucleons (the real part of the nucleon selfenergy modifies the free nucleon dispersion relation, while the imaginary part takes into account two nucleon absorption reaction channels). The model has no free parameters. The $W^{ \pm} N$ couplings and form factors are fixed in the vacuum, while the main features concerning the nuclear corrections, expansion parameter and all sorts of constants, are completely fixed from previous hadron-nucleus studies (pionic atoms, elastic and inelastic pion-nucleus reactions, $\Lambda$ - hypernuclei, etc.) [41-43]. Thus, the model is a natural extension of previous studies [41-45] on electron, photon and pion dynamics in nuclei, and should be able to describe inclusive CC QE neutrino and antineutrino nuclear reactions at intermediate energies of interest for future neutrino oscillation experiments. Even though the scarce existing CC data involve very low nuclear excitation energies, for which specific details of the nuclear structure might play an important role, the model of Ref. [34] provides one of the best existing combined description of the inclusive muon capture in ${ }^{12} \mathrm{C}$ and of the measurements of the ${ }^{12} \mathrm{C}$ $\left(\nu_{\mu}, \mu^{-}\right) X$ and ${ }^{12} \mathrm{C}\left(\nu_{e}, e^{-}\right) X$ reactions near threshold. Inclusive muon capture from other nuclei is also successfully described by the model. Besides, above, let us say 80 or $100 \mathrm{MeV}$ of energy transferred to the nucleus, this many body framework leads also to excellent results for the $\left(e, e^{\prime}\right)$ inclusive reaction in nuclei, not only in the $\mathrm{QE}$ region, but also when it is extended to the study of the $\Delta$-peak and the dip region (situated between the QE and the $\Delta$ peaks) $[44]^{3}$ and to the description of the absorption of real photons by nuclei [45].

In Sect. II, we extend the model of Ref. [34] to NC driven processes, both for neutrino and antineutrino induced nuclear reactions in the QE region. Thus we compute, for a fixed incoming neutrino or antineutrino Laboratory (LAB) energy, the inclusive QE cross sections $d^{2} \sigma / d \Omega^{\prime} d E^{\prime}\left(\Omega^{\prime}, E^{\prime}\right.$ are the solid angle and energy of the outgoing lepton) for $\left(\nu_{l}, \nu_{l}\right),\left(\nu_{l}, l^{-}\right),\left(\bar{\nu}_{l}, \bar{\nu}_{l}\right)$ and $\left(\bar{\nu}_{l}, l^{+}\right)$processes. This cross section gives us the reaction probability and it is the first required ingredient to start with our cascade model to describe the collisions suffered by the nucleons through their way out of the nucleus ${ }^{4}$. Details on the MC simulation are given in Sect. III. The MC method used here was designed for

\footnotetext{
${ }^{2}$ For nucleon energies above $1 \mathrm{GeV}$, the Glauber model [39], which is a multiple-scattering extension of the eikonal approximation, has also been used (see for instance the recent work of Ref. [35]). In this approach, a relativistic plane wave is modulated by a factor which accounts for the absorption into unobserved channels.

3 Data in ${ }^{12} \mathrm{C},{ }^{40} \mathrm{Ca}$ and ${ }^{208} \mathrm{~Pb}$ of differential cross sections for different electron kinematics and split into longitudinal and transverse response functions are successfully described.

${ }^{4}$ Besides, we also compute differential cross sections with respect to $d^{3} r$. Thus, we also know the point of the nucleus where the gauge boson was absorbed, and we can start from there our MC propagation of the ejected nucleon.
} 
single and multiple nucleon and pion emission reactions induced by pions $[42,46]$ and has been successfully employed to describe inclusive $(\gamma, \pi),(\gamma, N),(\gamma, N N), \ldots,(\gamma, N \pi), \ldots[47,48],\left(e, e^{\prime} \pi\right),\left(e, e^{\prime} N\right),\left(e, e^{\prime} N N\right), \ldots,\left(e, e^{\prime} N \pi\right), \ldots[49]$ reactions in nuclei or the neutron and proton spectra from the decay of $\Lambda$ hypernuclei [50]. Thus, we are using a quite robust and well tested MC simulator.

In Sect. IV we discuss our results and the main conclusions of this work are outlined in Sect. V. We start presenting (Subsect. IV A) results for the inclusive QE NC cross sections for both neutrino and antineutrino beams in several nuclei and in the next subsection we show results for inclusive $\left(\nu_{l}, \nu_{l} N\right),\left(\nu_{l}, l^{-} N\right),\left(\bar{\nu}_{l}, \bar{\nu}_{l} N\right)$ and $\left(\bar{\nu}_{l}, l^{+} N\right)$ reactions in nuclei at low energies, obtained from our cascade model. Some preliminary results of this work were already presented in Ref. [51]. Finally, in the appendix we give explicit expressions for the NC nucleon tensor, both in the impulse approximation and when RPA corrections are taken into account.

To end this introduction, we would like to devote a few words on the applicability of the nuclear model used here. One might think that a LFG description of the nucleus is poor, and that a proper finite nuclei treatment is necessary. For inclusive processes and nuclear excitation energies of around $100 \mathrm{MeV}$ or higher, the findings of Refs. [43], [44] and [45] clearly contradict this conclusion. The reason is that in these circumstances one should sum up over several nuclear configurations, both in the discrete and in the continuum, and this inclusive sum is almost not sensitive to the details of the nuclear wave function ${ }^{5}$, in sharp contrast to what happens in the case of exclusive processes where the final nucleus is left in a determined nuclear level. On the other hand, the LFG description of the nucleus allows for an accurate treatment of the dynamics of the elementary processes (interaction of gauge bosons with nucleons, nucleon resonances, and mesons, interaction between nucleons or between mesons and nucleons, etc.) which occur inside the nuclear medium. Within a finite nuclei scenario, such a treatment becomes hard to implement, and often the dynamics is simplified in order to deal with more elaborated nuclear wave functions. This simplification of the dynamics cannot lead to a good description of nuclear inclusive electroweak processes at the intermediate energies of interest for future neutrino experiments.

\section{EXTENSION OF THE MODEL OF REF. [34] TO NEUTRAL-CURRENTS}

\section{A. General formalism: hadronic tensor and many body expansion}

We will first focus on the neutrino induced inclusive reaction driven by the electroweak NC

$$
\nu_{l}(k)+A_{Z} \rightarrow \nu_{l}\left(k^{\prime}\right)+X
$$

and we will follow the same notation and convention as in Ref. [34]. The double differential cross section, with respect to the outgoing neutrino kinematical variables, for the process of Eq. (1) and for massless neutrinos, is given in the LAB frame by

$$
\frac{d^{2} \sigma_{\nu \nu}}{d \Omega\left(\hat{k}^{\prime}\right) d\left|\vec{k}^{\prime}\right|}=\frac{\left|\vec{k}^{\prime}\right|^{2} M_{i} G^{2}}{4 \pi^{2}}\left\{2 W_{1} \sin ^{2} \frac{\theta^{\prime}}{2}+W_{2} \cos ^{2} \frac{\theta^{\prime}}{2}-W_{3} \frac{|\vec{k}|+\left|\vec{k}^{\prime}\right|}{M_{i}} \sin ^{2} \frac{\theta^{\prime}}{2}\right\}
$$

with $\vec{k}$ and $\vec{k}^{\prime}$ the LAB neutrino momenta, $G=1.1664 \times 10^{-11} \mathrm{MeV}^{-2}$, the Fermi constant, $\theta^{\prime}$ the outgoing neutrino scattering angle and $M_{i}$ the target nucleus mass. To obtain Eq. (2) we have neglected the four-momentum carried out by the intermediate $Z$-boson with respect to its mass. Finally, the three independent, Lorentz scalar and real, structure functions, $W_{i}\left(q^{2}\right)$, enter into the definition of the hadronic tensor, $W^{\mu \nu}$, which includes all sort of nonleptonic vertices and corresponds to the neutral current electroweak transitions of the target nucleus, $i$, to all possible

\footnotetext{
5 The results of Ref. [34] for the inclusive muon capture in nuclei through the whole periodic table, where the capture widths vary from about $4 \times 10^{4} \mathrm{~s}^{-1}$ in ${ }^{12} \mathrm{C}$ to $1300 \times 10^{4} \mathrm{~s}^{-1}$ in ${ }^{208} \mathrm{~Pb}$, and of the LSND measurements of the ${ }^{12} \mathrm{C}\left(\nu_{\mu}, \mu^{-}\right) X$ and ${ }^{12} \mathrm{C}\left(\nu_{e}, e^{-}\right) X$ reactions near threshold indicate that the predictions of our scheme, for totally integrated inclusive observables, could even be extended to much smaller, of the order of 10 or $20 \mathrm{MeV}$, nuclear excitation energies. In this respect, the works of Refs. [52] and [53] for inclusive muon capture and radiative pion capture in nuclei, respectively, turn out to be quite enlightening. In those works, continuum shell model results are compared to those obtained from a LFG model for several nuclei from ${ }^{12} \mathrm{C}$ to ${ }^{208} \mathrm{~Pb}$. The differential decay width shapes predicted for the two set of models are substantially different. Shell model distributions present discrete contributions and in the continuum appear sharp scattering resonances. Despite the fact that those distinctive features do not appear in the LFG differential decay widths, the totally integrated widths (inclusive observable) obtained from both descriptions of the process do not differ in more than 5 or $10 \%$. The typical nuclear excitation energies in muon and radiative pion capture in nuclei are small, of the order of $20 \mathrm{MeV}$, and thus one expects that at higher excitation energies, where one should sum up over a larger number of nuclear final states, the LFG predictions for inclusive observables would become even more reliable.
} 
final states. It is thus given by (in our convention, we take $\epsilon_{0123}=+1$ and the metric $\left.g^{\mu \nu}=(+,-,-,-)\right)^{6}$

$$
\begin{aligned}
\frac{W^{\mu \sigma}}{2 M_{i}} & =\frac{1}{4 M_{i}^{2}} \overline{\sum_{f}}(2 \pi)^{3} \delta^{4}\left(P_{f}^{\prime}-P-q\right)\left\langle f\left|j_{\mathrm{nc}}^{\mu}(0)\right| i\right\rangle\left\langle f\left|j_{\mathrm{nc}}^{\sigma}(0)\right| i\right\rangle^{*} \\
& =-g^{\mu \nu} W_{1}+\frac{P^{\mu} P^{\nu}}{M_{i}^{2}} W_{2}+\mathrm{i} \frac{\epsilon^{\mu \nu \gamma \delta} P_{\gamma} q_{\delta}}{2 M_{i}^{2}} W_{3}+\frac{q^{\mu} q^{\nu}}{M_{i}^{2}} W_{4}+\frac{P^{\mu} q^{\nu}+P^{\nu} q^{\mu}}{2 M_{i}^{2}} W_{5}+\mathrm{i} \frac{P^{\mu} q^{\nu}-P^{\nu} q^{\mu}}{2 M_{i}^{2}} W_{6}
\end{aligned}
$$

with $P^{\mu}$ the four-momentum of the initial nucleus $\left(M_{i}^{2}=P^{2}\right), P_{f}^{\prime}$ the total four momentum of the hadronic state $f$ and $q=k-k^{\prime}$ the four momentum transferred to the nucleus. The bar over the sum denotes the average over initial spins. By construction, the hadronic tensor accomplishes

$$
W^{\mu \sigma}=W_{s}^{\mu \sigma}+\mathrm{i} W_{a}^{\mu \sigma}
$$

with $W_{s}^{\mu \sigma}\left(W_{a}^{\mu \sigma}\right)$ real symmetric (antisymmetric) tensors, and finally for the NC we take

$$
j_{\mathrm{nc}}^{\mu}=\bar{\Psi}_{u} \gamma^{\mu}\left(1-\frac{8}{3} \sin ^{2} \theta_{W}-\gamma_{5}\right) \Psi_{u}-\bar{\Psi}_{d} \gamma^{\mu}\left(1+\frac{4}{3} \sin ^{2} \theta_{W}-\gamma_{5}\right) \Psi_{d}-\bar{\Psi}_{s} \gamma^{\mu}\left(1+\frac{4}{3} \sin ^{2} \theta_{W}-\gamma_{5}\right) \Psi_{s}
$$

with $\Psi_{u}, \Psi_{d}$ and $\Psi_{s}$ quark fields, and $\theta_{W}$ the Weinberg angle $\left(\sin ^{2} \theta_{W}=0.231\right)$.

Taking $\vec{q}$ in the $z$ direction, ie, $\vec{q}=|q| \vec{u}_{z}$, and $P^{\mu}=\left(M_{i}, \overrightarrow{0}\right)$, it is straightforward to find the six structure functions in terms of the $W^{00}, W^{x x}=W^{y y}, W^{z z}, W^{x y}$ and $W^{0 z}$ components of the hadronic tensor ${ }^{7}$. The neutrino cross section, Eq. (2), does not depend on $M_{i}$, as can be seen from the relations of Eq. (6), and also note that the structure functions $W_{4,5,6}$ do not contribute in the limit of massless neutrinos.

The cross section for the antineutrino induced nuclear reaction

$$
\bar{\nu}_{l}(k)+A_{Z} \rightarrow \bar{\nu}_{l}\left(k^{\prime}\right)+X
$$

is easily obtained from Eq. (2), just by changing the sign of the parity-violating term $\left(W_{3}\right)$.

The hadronic tensor is determined by the $Z^{0}$-boson selfenergy, $\Pi_{Z}^{\mu \rho}(q)$, in the nuclear medium. We follow here the formalism of Ref. [34], and we evaluate the selfenergy, $\Sigma_{\nu}^{r}(k ; \rho)$, of a neutrino, with four-momentum $k$ and helicity $r$, moving in infinite nuclear matter of constant density $\rho$. We find,

$$
\begin{aligned}
& W_{s}^{\mu \sigma}=-\Theta\left(q^{0}\right)\left(\frac{4 \cos \theta_{W}}{g}\right)^{2} \int \frac{d^{3} r}{2 \pi} \operatorname{Im}\left[\Pi_{Z}^{\mu \sigma}+\Pi_{Z}^{\sigma \mu}\right](q ; \rho(r)) \\
& W_{a}^{\mu \sigma}=-\Theta\left(q^{0}\right)\left(\frac{4 \cos \theta_{W}}{g}\right)^{2} \int \frac{d^{3} r}{2 \pi} \operatorname{Re}\left[\Pi_{Z}^{\mu \sigma}-\Pi_{Z}^{\sigma \mu}\right](q ; \rho(r)),
\end{aligned}
$$

where we have used the Local Density Approximation (LDA) to obtain results in a finite nucleus of density $\rho(r)$, and $g$ is the gauge weak coupling constant, $g=e / \sin \theta_{W}$, related to the Fermi constant: $G / \sqrt{2}=g^{2} / 8 M_{W}^{2}$, with $e$ the electron charge.

As we see, the basic object is the selfenergy of the Gauge Boson $\left(Z^{0}\right)$ inside of the nuclear medium. As it is done in Ref. [44] for electro induced nuclear reactions, we plan to perform a many body expansion, where the relevant gauge boson absorption modes would be systematically incorporated: absorption by one nucleon, or a pair of nucleons or even three nucleon mechanisms, real and virtual meson $(\pi, \rho, \cdots)$ production, excitation of $\Delta$ of higher resonance degrees of freedom, etc. In addition, nuclear effects such as RPA or SRC should also be taken into account. Some of the $Z^{0}$-absorption modes are depicted in Fig. 1.

\footnotetext{
${ }^{6}$ Note that: (i) Eq. (3) holds with states normalized so that $\left\langle\vec{p} \mid \vec{p}^{\prime}\right\rangle=(2 \pi)^{3} 2 p_{0} \delta^{3}\left(\vec{p}-\vec{p}^{\prime}\right)$, (ii) the sum over final states $f$ includes an integration $\int \frac{d^{3} p_{j}}{(2 \pi)^{3} 2 E_{j}}$, for each particle $j$ making up the system $f$, as well as a sum over all spins involved.

7 Thus, one readily finds
}

$$
W_{1}=\frac{W^{x x}}{2 M_{i}}, \quad W_{2}=\frac{1}{2 M_{i}}\left(W^{00}+W^{x x}+\frac{\left(q^{0}\right)^{2}}{|\vec{q}|^{2}}\left(W^{z z}-W^{x x}\right)-2 \frac{q^{0}}{|\vec{q}|} \operatorname{Re} W^{0 z}\right), \quad W_{3}=-\mathrm{i} \frac{W^{x y}}{|\vec{q}|}
$$




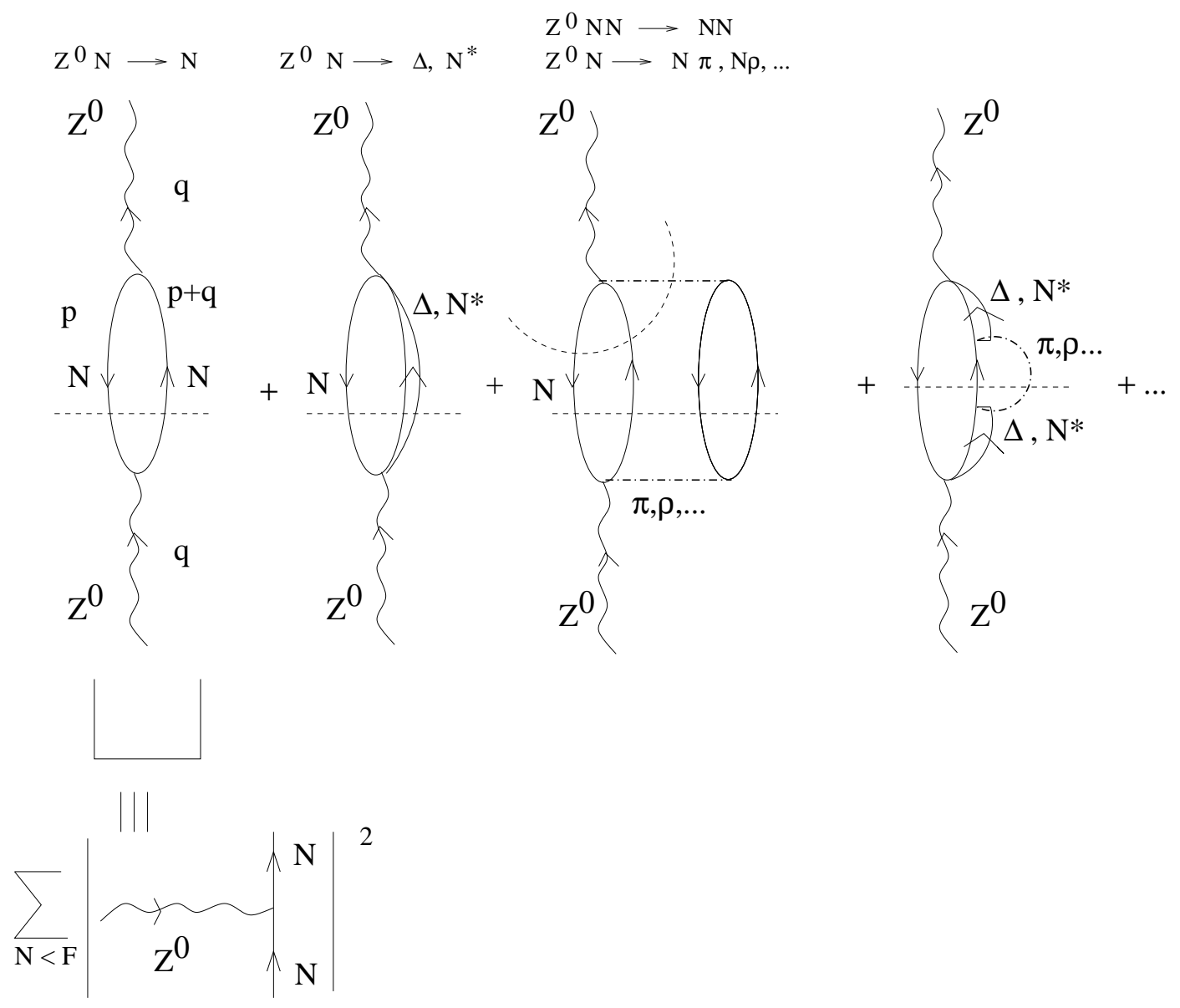

FIG. 1: Diagrammatic representation of some diagrams contributing to the $Z^{0}$-selfenergy.

\section{B. QE contribution and Pauli blocking}

The virtual $Z^{0}$ can be absorbed by one nucleon leading to the QE contribution of the nuclear response function. Such a contribution corresponds to a $1 \mathrm{p} 1 \mathrm{~h}$ nuclear excitation (first of the diagrams depicted in Fig. 1). To evaluate this selfenergy, the free nucleon propagator in the medium is required.

$$
S(p ; \rho)=(\not p+M) G(p ; \rho), \quad G(p ; \rho)=\left(\frac{1}{p^{2}-M^{2}+\mathrm{i} \epsilon}+\frac{2 \pi \mathrm{i}}{2 E(\vec{p})} \delta\left(p^{0}-E(\vec{p})\right) \Theta\left(k_{F}-|\vec{p}|\right)\right)
$$

with the local Fermi momentum $k_{F}(r)=\left(3 \pi^{2} \rho(r) / 2\right)^{1 / 3}, M=940 \mathrm{MeV}$ the nucleon mass, and $E(\vec{p})=\sqrt{M^{2}+\vec{p}^{2}}$. We will work on a non-symmetric nuclear matter with different Fermi sea levels for protons, $k_{F}^{p}$, than for neutrons, $k_{F}^{n}$ (equation above, but replacing $\rho / 2$ by $\rho_{p}$ or $\rho_{n}$, with $\rho=\rho_{p}+\rho_{n}$ ). On the other hand, for the $Z^{0} N N$ vertex we take $(N=n$ or $p)$

$$
<N ; \vec{p}^{\prime}=\vec{p}+\vec{q}\left|j_{n c}^{\alpha}(0)\right| N ; \vec{p}>=\bar{u}\left(\vec{p}^{\prime}\right)\left(V_{N}^{\alpha}-A_{N}^{\alpha}\right) u(p)
$$

with spinor normalization given by $\bar{u} u=2 m$, and vector and axial nucleon currents given by

$$
V_{N}^{\alpha}=2 \times\left(F_{1}^{Z}\left(q^{2}\right) \gamma^{\alpha}+i \mu_{Z} \frac{F_{2}^{Z}\left(q^{2}\right)}{2 M} \sigma^{\alpha \nu} q_{\nu}\right)_{N}, \quad A_{N}^{\alpha}=\left(G_{A}^{Z}\left(q^{2}\right) \gamma^{\alpha} \gamma_{5}+G_{P}^{Z}\left(q^{2}\right) q^{\alpha} \gamma_{5}\right)_{N}
$$

Invariance under G-parity has been assumed to discard a term of the form $\left(p^{\mu}+p^{\prime \mu}\right) \gamma_{5}$ in the axial sector, while invariance under time reversal guarantees that all form factors are real. Besides, and thanks to $\mathrm{SU}(3)$ symmetry, some relations exist among the $\mathrm{NC}$ form factors and the $\mathrm{CC}\left(F_{1,2}^{V}, G_{A}\right.$ and $G_{P}$, see Ref. [34]) and the electromagnetic 
$\operatorname{ones}^{8}\left(F_{1}^{p, n}, \mu_{p} F_{2}^{p}\right.$ and $\left.\mu_{n} F_{2}^{n}\right)$

$$
\begin{aligned}
\left(F_{1}^{Z}\right)^{p, n} & = \pm F_{1}^{V}-2 \sin ^{2} \theta_{W} F_{1}^{p, n}-\frac{1}{2} F_{1}^{s} \\
\left(\mu_{Z} F_{2}^{Z}\right)^{p, n} & = \pm \mu_{V} F_{2}^{V}-2 \sin ^{2} \theta_{W} \mu_{p, n} F_{2}^{p, n}-\frac{1}{2} \mu_{s} F_{2}^{s} \\
\left(G_{A, P}^{Z}\right)^{p, n} & = \pm G_{A, P}-G_{A, P}^{s}
\end{aligned}
$$

where $F_{1}^{s}, \mu_{s} F_{2}^{s}, G_{A}^{s}$ and $G_{P}^{s}$ are the strange vector and axial nucleon form factors [55]. The pseudoscalar part of the axial current does not contribute to the differential cross section for massless neutrinos and for the rest of strange form factors we use the results of the fit II of Ref. [56],

$$
G_{A}^{s}\left(q^{2}\right)=\frac{g_{A}^{s}}{\left(1-q^{2} /\left(M_{A}^{s}\right)^{2}\right)^{2}}, \quad F_{1}^{s}\left(q^{2}\right)=\mu_{s} F_{2}^{s}\left(q^{2}\right)=0
$$

with $g_{A}^{s}=-0.15$ and $M_{A}^{s}=1049 \mathrm{MeV}$. With all of these ingredients is straightforward to evaluate the contribution to the $Z^{0}$-selfenergy of the first diagram of Fig. 1, which leads to

$$
\begin{aligned}
W^{\mu \nu}\left(q^{0}, \vec{q}\right) & =-\frac{1}{2 M^{2}} \int_{0}^{\infty} d r r^{2}\left\{2 \Theta\left(q^{0}\right) \int \frac{d^{3} p}{(2 \pi)^{3}} \frac{M}{E(\vec{p})} \frac{M}{E(\vec{p}+\vec{q})}(-\pi)\right. \\
& \times \sum_{N=n, p}\left\{\Theta\left(k_{F}^{N}(r)-|\vec{p}|\right) \Theta\left(|\vec{p}+\vec{q}|-k_{F}^{N}(r)\right) A_{N}^{\nu \mu}(p, q)\right\}_{p^{0}=E(\vec{p})} \delta\left(q^{0}-Q+E(\vec{p})-E(\vec{p}+\vec{q})\right)
\end{aligned}
$$

where $Q$ is the experimental $Q$-value, included in order to properly reproduce the energy threshold. For inclusive observables we have set it to zero to approximately take into account the possibility of elastic scattering and transitions to nuclear excited states. The $d^{3} p$ integrations above can be analytically done and all of them are determined by the imaginary part of the relativistic Lindhard function, $\bar{U}_{R}\left(q, k_{F}^{N}, k_{F}^{N}\right)$. Explicit expressions can be found in Appendix B of Ref. [34]. The NC nucleon tensor, $A_{N}^{\nu \mu}$, can be found in Appendix A 1. The non-relativistic reduction of the hadronic tensor can be obtained by replacing the factors $M / E(\vec{p})$ and $M / E(\vec{p}+\vec{q})$ in Eq. (17) by one. Explicit expressions can be found in Appendix C of Ref. [34].

To finish this section, we devote a few words to the Low Density Theorem (LDT). At low nuclear densities the imaginary part of the relativistic Lindhard function can be approximated by

$$
\operatorname{Im} \bar{U}_{R}\left(q, k_{F}^{N}, k_{F}^{N}\right) \approx-\pi \rho_{N} \frac{M}{E(\vec{q})} \delta\left(q^{0}+M-E(\vec{q})\right)
$$

and thus one readily finds

$$
\sigma_{\nu_{l}+A_{Z} \rightarrow \nu_{l}+X} \approx N \sigma_{\nu_{l}+n \rightarrow \nu_{l}+n}+Z \sigma_{\nu_{l}+p \rightarrow \nu_{l}+p}, \quad N=A-Z
$$

which agrees with the LDT.

\section{QE contribution: RPA nuclear correlation and FSI effects}

Pauli blocking, through the imaginary part of the Lindhard function, is the main nuclear effect included in the hadronic tensor of Eq. (17). RPA nuclear correlation and FSI effects played a crucial role for inclusive QE CC neutrino-nucleus reactions, and we include those here, following the same formalism as in Ref. [34].

\section{1. $R P A$}

We replace the $1 \mathrm{p} 1 \mathrm{~h}$ contribution to the $Z^{0}$ selfenergy by an RPA response as shown diagrammatically in Fig. 2. For that purpose we use an effective ph-ph interaction of the Landau-Migdal type, with explicit contribution of pion and rho meson exchanges,

$$
V=c_{0}\left\{f_{0}(\rho)+f_{0}^{\prime}(\rho) \vec{\tau}_{1} \vec{\tau}_{2}+g_{0}(\rho) \vec{\sigma}_{1} \vec{\sigma}_{2}\right\}+\vec{\tau}_{1} \vec{\tau}_{2}\left\{V_{l}(q) \vec{\sigma}_{1} \hat{q} \vec{\sigma}_{2} \hat{q}+V_{t}(q)\left(\vec{\sigma}_{1} \vec{\sigma}_{2}-\vec{\sigma}_{1} \hat{q} \vec{\sigma}_{2} \hat{q}\right)\right\}
$$

\footnotetext{
${ }^{8}$ We use the parameterization of Galster and collaborators [54], which is also compiled in Ref. [34].
} 
where $\vec{\sigma}$ and $\vec{\tau}$ are Pauli matrices acting on the nucleon spin and isospin spaces, respectively, and $\hat{q}=\vec{q} /|\vec{q}|$. We take the coefficients $f_{0}, f_{0}^{\prime}$ and $g_{0}$ from Ref. [57],

$$
f_{i}(\rho(r))=\frac{\rho(r)}{\rho(0)} f_{i}^{(i n)}+\left[1-\frac{\rho(r)}{\rho(0)}\right] f_{i}^{(e x)}
$$

where

$$
\begin{aligned}
& f_{0}^{(i n)}=0.07 \\
& f_{0}^{(e x)}=-2.15 \\
& g_{0}^{(i n)}=g_{0}^{(e x)}=g_{0}=0.575
\end{aligned}
$$

and $c_{0}=380 \mathrm{MeVfm}^{3}$. In the $S=1=T$ channel $(\vec{\sigma} \vec{\sigma} \vec{\tau} \vec{\tau}$ operator) we use an interaction with explicit $\pi$ (longitudinal) and $\rho$ (transverse) exchanges, which has been used for the renormalization of the pionic and pion related channels in different nuclear reactions at intermediate energies [42]-[45]. The strengths of the ph-ph interaction in the longitudinal and transverse channel are given by

$$
V_{l}\left(q^{0}, \vec{q}\right)=\frac{f^{2}}{m_{\pi}^{2}}\left\{\left(\frac{\Lambda_{\pi}^{2}-m_{\pi}^{2}}{\Lambda_{\pi}^{2}-q^{2}}\right)^{2} \frac{\vec{q}^{2}}{q^{2}-m_{\pi}^{2}}+g_{l}^{\prime}(q)\right\}, \quad \frac{f^{2}}{4 \pi}=0.08, \quad \Lambda_{\pi}=1200 \mathrm{MeV}
$$
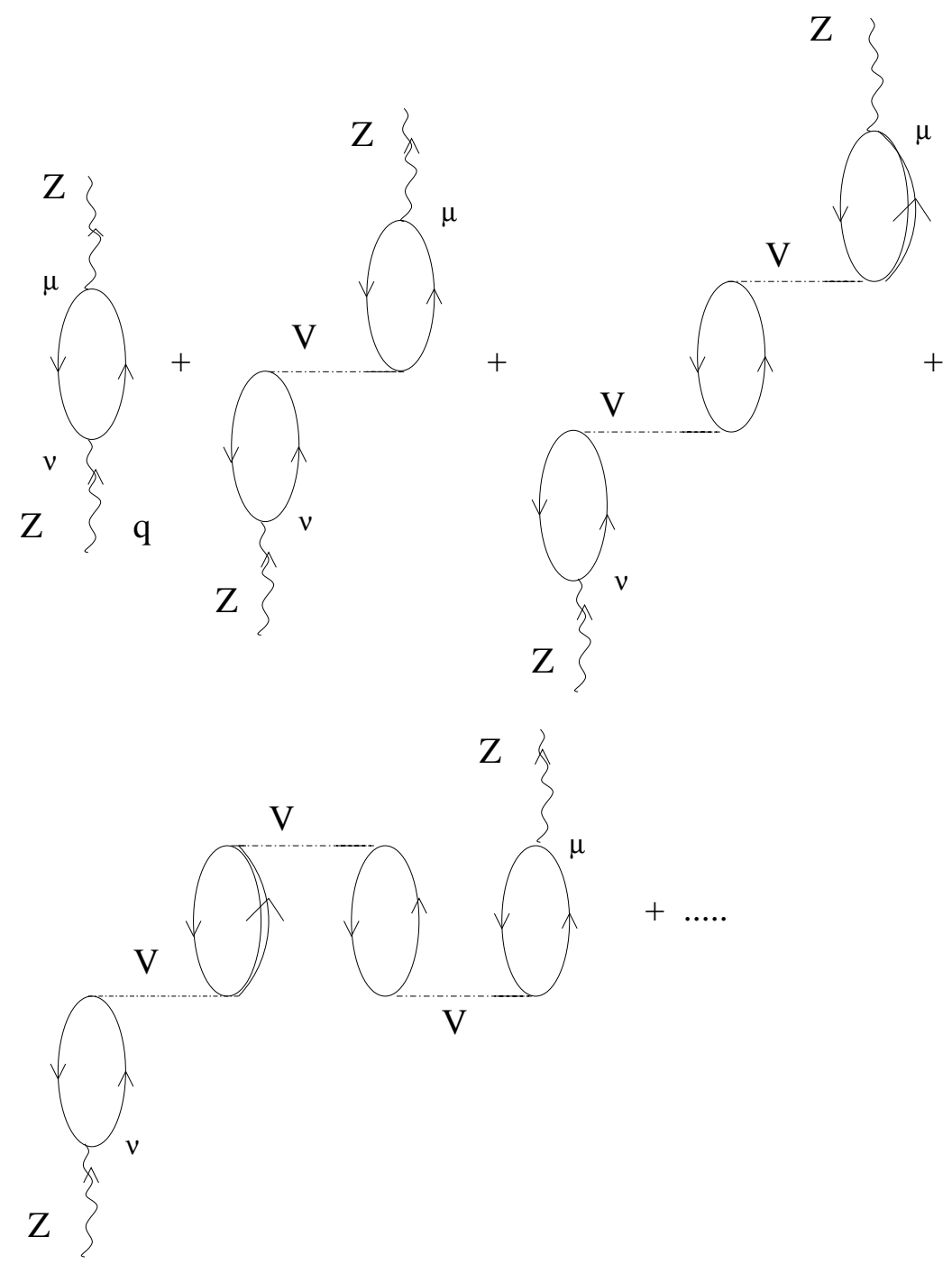

FIG. 2: Set of irreducible diagrams responsible for the polarization (RPA) effects in the 1 p1h contribution to the $Z$-selfenergy. 


$$
V_{t}\left(q^{0}, \vec{q}\right)=\frac{f^{2}}{m_{\pi}^{2}}\left\{C_{\rho}\left(\frac{\Lambda_{\rho}^{2}-m_{\rho}^{2}}{\Lambda_{\rho}^{2}-q^{2}}\right)^{2} \frac{\vec{q}^{2}}{q^{2}-m_{\rho}^{2}}+g_{t}^{\prime}(q)\right\}, \quad C_{\rho}=2, \quad \Lambda_{\rho}=2500 \mathrm{MeV}, \quad m_{\rho}=770 \mathrm{MeV}
$$

The SRC functions $g_{l}^{\prime}$ and $g_{t}^{\prime}$ have a smooth $q$-dependence $[41,58]$, which we will not consider here ${ }^{9}$, and thus we will take $g_{l}^{\prime}(q)=g_{t}^{\prime}(q)=g^{\prime}=0.63$ as it was done in the study of inclusive nuclear electron scattering carried out in Ref. [44], and also in some of the works of Ref. [42, 43].

The above interaction has been successfully tested in different nuclear processes at intermediate energies [41]-[49], and we will use here the same form and parameters as in our recent study of CC neutrino nucleus reactions [34]. As it is explained there, $\Delta(1232)$ degrees of freedom are also taken into account. Given the spin-isospin quantum numbers of the $\Delta$ resonance, these degrees of freedom only modify the vector-isovector $(S=1=T)$ channel of the RPA response function. The ph- $\Delta \mathrm{h}$ and $\Delta \mathrm{h}-\Delta \mathrm{h}$ effective interactions are obtained from the interaction of Eq. (20) by replacing $\vec{\sigma} \rightarrow \vec{S}, \vec{\tau} \rightarrow \vec{T}$, where $\vec{S}, \vec{T}$ are the spin, isospin $N \Delta$ transition operators [41] and $f \rightarrow f^{*}=2.13 f$, for any $\Delta$ which replaces a nucleon.

Thus, the $V$ lines in Fig. 2 stand for the effective $\operatorname{ph}(\Delta \mathrm{h})-\operatorname{ph}(\Delta \mathrm{h})$ interaction described so far. Keeping track of the operators responsible, we have examined and renormalized all different contributions to the NC nucleon tensor $A_{N}^{\mu \nu}$, by summing up the RPA series depicted in Fig. 2. The procedure is discussed in detail in Ref. [34], now however the isospin structure of the $Z^{0} N N$ vertex

$$
\Gamma^{\mu}\left(F_{p} \frac{1+\tau_{z}}{2}+F_{n} \frac{1-\tau_{z}}{2}\right)
$$

with $\Gamma^{\mu}$ some matrix in the Dirac space and $F$ some form factor, does not select only the isovector channels of the in medium effective $\operatorname{ph}(\Delta \mathrm{h})-\mathrm{ph}(\Delta \mathrm{h})$ interaction, as it is the case for the CC induced processes studied in [34], and hence we also find contributions from the isoscalar part of the effective force.

For simplicity and to compute these polarization corrections, we have assumed a symmetric nuclear matter with the same Fermi sea level for protons and neutrons. On the other hand, since the $\mathrm{ph}(\Delta \mathrm{h})-\mathrm{ph}(\Delta \mathrm{h})$ effective interaction is non-relativistic, we have computed polarization effects only for the leading and next-to-leading terms in the $p / M$ expansion. Thus, order $\mathcal{O}\left(k_{F} \vec{p}^{2} / M^{2}, k_{F} \vec{p}^{\prime 2} / M^{2}, k_{F} q^{0} / M\right)$ has been neglected. We have made an exception to the above rule, and since $\mu_{Z}$ could be relatively large, we have taken $\mu_{Z} F_{2}^{Z}|\vec{q}| / M$ to be of order $\mathcal{O}(0)$ in the $p / M$ expansion. With all these ingredients, we find $\delta W_{\mathrm{RPA}}^{\mu \nu}$, contribution which has to be added to the hadronic tensor, $W^{\mu \nu}$, given in Eq. (17) to account for the medium polarization effects,

$$
\begin{aligned}
\delta W_{\mathrm{RPA}}^{\mu \nu} & =-\frac{1}{2 M^{2}} \int_{0}^{\infty} d r r^{2}\left\{2 \Theta\left(q^{0}\right) \int \frac{d^{3} p}{(2 \pi)^{3}} \frac{M}{E(\vec{p})} \frac{M}{E(\vec{p}+\vec{q})}(-\pi) \delta\left(q^{0}+E(\vec{p})-E(\vec{p}+\vec{q})\right)\right. \\
& \times\left\{\Theta\left(k_{F}(r)-|\vec{p}|\right) \Theta\left(|\vec{p}+\vec{q}|-k_{F}(r)\right) \delta A_{\mathrm{RPA}}^{\nu \mu}(p, q)\right\}_{p^{0}=E(\vec{p})}
\end{aligned}
$$

The $00,0 z, z z, x x$ and $x y$ components of the RPA contribution to the NC nucleon tensor, $\delta A_{\mathrm{RPA}}^{\nu \mu}(p, q)$, are given in Sect. A 2 of the Appendix. There, the density dependent tensor $\delta A_{\mathrm{RPA}}^{\nu \mu}(p, q)$ is given in terms of the parameters of the effective $\operatorname{ph}(\Delta \mathrm{h})-\operatorname{ph}(\Delta \mathrm{h})$ force and $U\left(q, k_{F}\right)=U_{N}+U_{\Delta}$, the Lindhard function of Ref. [58], which for simplicity we evaluate $^{10}$ in an isospin symmetric nuclear medium of density $\rho$. The different couplings for $N$ and $\Delta$ are incorporated in $U_{N}$ and $U_{\Delta}$ and then the same interaction strengths $V_{l}$ and $V_{t}$ are used for ph and $\Delta$ h excitations [41]-[49]. Note that, backward (crossed term of the Lindhard function) propagating ph and $\Delta h$ excitations are also taken into account within our framework. For positive values of $q^{0}$ the backward propagating ph excitation has no imaginary part, and for QE kinematics $U_{\Delta}$ is also real.

Finally, we should stress that the $f_{0}^{\prime}, f_{0}$ and $g_{0}$ terms of the effective interaction cannot produce $\Delta$ h excitations and therefore, when these terms are involved in the RPA renormalization, only the nucleon Lindhard function $\left(U_{N}\right)$ appears (see Eqs. (A9)).

\section{FSI: dressed nucleon propagators in the nuclear medium}

Once a ph excitation is produced by the virtual $Z^{0}$-boson, the nucleon is interacting with the rest of nucleons of the nucleus, colliding many times, thus inducing the emission of other nucleons. The result of it is a quenching of the

\footnotetext{
9 This is justified because taking into account the $q$-dependence leads to minor changes for low and intermediate energies and momenta, where this effective ph-ph interaction should be used.

10 The functions $U_{N}$ and $U_{\Delta}$ are defined in Eqs.(2.9) and (3.4) of Ref. [58], respectively.
} 
QE peak respect to the simple ph excitation calculation and a spreading of the strength, or widening of the peak. In our many body scheme we will account for the FSI by using nucleon propagators properly dressed with a realistic selfenergy in the medium, which depends explicitly on the energy and the momentum [59]. Hence we substitute the particle nucleon propagator, $G(p ; \rho)$, in Eq. (10) by a renormalized nucleon propagator, $G_{\mathrm{FSI}}(p ; \rho)$, including the nucleon selfenergy in the medium, $\Sigma\left(p^{0}, \vec{p} ; \rho\right)$,

$$
G_{\mathrm{FSI}}(p ; \rho)=\frac{1}{p^{0}-\bar{E}(\vec{p})-\Sigma\left(p^{0}, \vec{p} ; \rho\right)}
$$

with $\bar{E}(\vec{p})=M+\vec{p}^{2} / 2 M$. This approach led to excellent results in the study of inclusive electron and CC neutrino scattering from nuclei [34, 44]. Since the model of Ref. [59] is not Lorentz relativistic and it also considers an isospin symmetric nuclear medium, we will only discuss the FSI effects for nuclei with approximately equal number of protons and neutrons, and using non-relativistic kinematics for the nucleons. Thus, we have obtained Eq. (26) from the non-relativistic reduction of $G(p ; \rho)$, in Eq. (10), by including the nucleon selfenergy.

To account for FSI effects in an isospin symmetric nuclear medium of density $\rho$ we should make the following substitution $[34,44]$

$$
\begin{aligned}
& \left.2 \Theta\left(q^{0}\right) \int \frac{d^{3} p}{(2 \pi)^{3}} \Theta\left(k_{F}(r)-|\vec{p}|\right) \Theta\left(|\vec{p}+\vec{q}|-k_{F}(r)\right)(-\pi) \delta\left(q^{0}+\bar{E}(\vec{p})-\bar{E}(\vec{p}+\vec{q})\right) \mathcal{A}^{\nu \mu}(p, q)\right|_{p^{0}=\bar{E}(\vec{p})} \\
\rightarrow & -\left.\frac{\Theta\left(q^{0}\right)}{4 \pi^{2}} \int d^{3} p \int_{\mu-q^{0}}^{\mu} d \omega S_{h}(\omega, \vec{p} ; \rho) S_{p}\left(q^{0}+\omega, \vec{p}+\vec{q} ; \rho\right) \mathcal{A}^{\nu \mu}(p, q)\right|_{p^{0}=\bar{E}(\vec{p})}, \quad \mathcal{A}^{\mu \nu}=A_{N}^{\mu \nu}, \delta A_{\mathrm{RPA}}^{\mu \nu}
\end{aligned}
$$

in the expression of the hadronic tensor (Eqs. (17) and (25)). $S_{h}, S_{p}$ are the hole and particle spectral functions related to nucleon selfenergy $\Sigma$ by means of

$$
S_{p, h}(\omega, \vec{p} ; \rho)=\mp \frac{1}{\pi} \frac{\operatorname{Im} \Sigma(\omega, \vec{p} ; \rho)}{[\omega-\bar{E}(\vec{p})-\operatorname{Re} \Sigma(\omega, \vec{p} ; \rho)]^{2}+[\operatorname{Im} \Sigma(\omega, \vec{p} ; \rho)]^{2}}
$$

with $\omega \geq \mu$ or $\omega \leq \mu$ for $S_{p}$ and $S_{h}$, respectively. The chemical potential $\mu$ is determined by

$$
\mu=M+\frac{k_{F}^{2}}{2 M}+\operatorname{Re} \Sigma\left(\mu, k_{F}\right)
$$

The $d^{3} p$ integrations have to be done now numerically, and since the imaginary part of the nucleon selfenergy for the hole states is much smaller than that of the particle states at intermediate nuclear excitation energies, we make the approximation of setting to zero $\operatorname{Im} \Sigma$ for the hole states. Thus, we take

$$
S_{h}(\omega, \vec{p} ; \rho)=\delta(\omega-\hat{E}(\vec{p})) \Theta(\mu-\hat{E}(p))
$$

where $\hat{E}(p)$ is the energy associated to a momentum $\vec{p}$ obtained self consistently by means of the equation

$$
\hat{E}(\vec{p})=\bar{E}(\vec{p})+\operatorname{Re} \Sigma(\hat{E}(\vec{p}), \vec{p} ; \rho)
$$

The same approximation is also used in some of the calculations for the particle spectral function. The effects of this approximation will be discussed in Sect. IV B.

\section{THE MONTE CARLO SIMULATION}

\section{A. Kinematics of the outgoing nucleon in the first step}

In a previous work [34] and in Sect. II we carried out a thorough evaluation of the CC and NC inclusive neutrino and antineutrino induced nuclear reactions in the QE region. Thus we have determined, for a fixed incoming neutrino or antineutrino LAB energy, the inclusive QE cross section $d^{2} \sigma / d \Omega^{\prime} d E^{\prime}\left(\Omega^{\prime}, E^{\prime}\right.$ are the solid angle and energy of the outgoing lepton). The absorption of the gauge boson $\left(W^{ \pm}\right.$or $\left.Z^{0}\right)$ with four momentum $q^{\mu}$ by one nucleon constitutes the reaction mechanism $(1 \mathrm{p} 1 \mathrm{~h} \text { excitation })^{11}$, and the corresponding reaction probability is determined by $d^{2} \sigma / d \Omega^{\prime} d E^{\prime}$.

\footnotetext{
11 This is not entirely correct, since two nucleon absorption modes are also considered, when the FSI effects described in Sect.II C 2 are taken into account. We will discuss this point later in Subsect. IV B.
} 
Moreover, within our scheme, we obtain $d^{2} \sigma / d \Omega^{\prime} d E^{\prime}$ after performing an integration over the whole nuclear volume (see Eqs. (16) and (17) of Ref. [34] and Sect. II, respectively). Thus, for a fixed transferred four momentum $q^{\mu}$, chosen according to $d^{2} \sigma / d \Omega^{\prime} d E^{\prime}$, we can randomly select the point of the nucleus where the absorption takes place using the profile $d^{5} \sigma / d \Omega^{\prime} d E^{\prime} d^{3} r$ evaluated in Ref. [34] for CC and in Sect. II for NC.

Now, we need on top of that to have the distribution of three-momenta of the outgoing nucleon. This can be done by not performing the integration over the three momentum, $\vec{p}$, of the nucleon occupied states in [34] and in Sect. II (see for instance Eq. (17) for the the NC case), since the outgoing nucleon momentum is $\vec{p}+\vec{q}$. However, in order to incorporate the nucleon scattering with other nucleons, we choose the alternative procedure of generating events probabilistically one by one, with a weight given by $d^{5} \sigma / d \Omega^{\prime} d E^{\prime} d^{3} r$. For each event in a certain position $\vec{r}$ and with a transferred four momentum $q^{\mu}$ we generate a random momentum $\vec{p}$ from the local Fermi sea. The vector $\vec{p}+\vec{q}$ gives us the direction of the nucleon. The energy of the nucleon is then obtained by imposing energy conservation assuming in this step and throughout all the MC simulation that the nucleons move in a Fermi gas under an attractive potential equal to the local Fermi energy and therefore

$$
\tilde{E}^{\prime}=\tilde{E}(\vec{p})+q^{0}
$$

where $\tilde{E}(\vec{p})=\sqrt{\vec{p}^{2}+M^{2}}-k_{F}^{2}(r) / 2 M$. This will provides us with the modulus of the outgoing momentum ${ }^{12} \vec{p}^{\prime}$. If it happens that $\left|\vec{p}^{\prime}\right|<k_{F}(r)$ (the local Fermi momentum) then the event is Pauli blocked, it is dismissed and another event is generated. Thus, we have already the configuration of the final state after the first step, namely, one nucleon produced in the point $\vec{r}$ of the nucleus with momentum $\vec{p}^{\prime}$. With respect to having a proton or a neutron in the final state, this is trivially done: for the $\mathrm{CC}$ case, the outgoing nucleon is a proton (neutron) for neutrino (antineutrino) induced processes, while for NC, in Sect. II, the reaction probability was already split into a proton and a neutron induced ones (see for instance Eq. (17) $)^{13}$.

\section{B. Nucleon propagation}

The nucleons in the nucleus move under the influence of a complex optical potential. The imaginary part of the potential is related to the probability of nucleon quasielastic collisions in the nucleus (and extra pion production at higher energies, which we do not consider here). We consider explicitly these collisions since they generate new nucleons going outside the nucleus. As with respect to the real part, we use it to determine the classical trajectories that the nucleons follow in the nucleus between collisions.

As done in Refs. $[48,49]$ we take as the real part of the nucleon-nucleus potential

$$
V(r)=V_{\infty}-\mathcal{E}(r)=-\frac{k_{F}^{2}}{2 M}=-\frac{1}{2 M}\left(\frac{3}{2} \pi^{2} \rho(r)\right)^{2 / 3}
$$

It represents the interaction of a single nucleon with the average potential due to the rest of the nucleons. This choice of $V(r)$ means that the total nucleon energy is the difference between its kinetic energy and the Fermi energy, $k_{F}^{2} / 2 M$.

With respect to collisions in our MC simulation we follow each excited nucleon by letting it move a short distance $d$ such that $P d<<1$ ( $P$ represents the probability per unit of length for a quasielastic collision ). The new position $\left(\vec{r}^{\prime}\right)$ and momentum $\left(\vec{p}^{\prime}\right)$ are taken from the Hamiltonian equations as

$$
\begin{aligned}
& \vec{r}^{\prime}=\vec{r}+\delta \vec{r}=\vec{r}+\frac{\vec{p}}{|\vec{p}|} d \\
& \vec{p}^{\prime}=\vec{p}+\delta \vec{p} ; \delta \vec{p}=-\frac{\partial V}{\partial r} \frac{E(p) d}{p} \frac{\vec{r}}{|\vec{r}|}
\end{aligned}
$$

which follow from the Hamilton equations or equivalently from energy and angular momentum conservation.

Our code selects randomly, according to the reaction probabilities which will be discussed in Subsect. III C, if the nucleon is scattered or not and, in the case of scattering, what kind of process takes place. If no collision takes place, we move the nucleon again. When the nucleon leaves the nucleus we stop the process and it is counted as a contribution to the cross section. If a $N N$ scattering is selected instead, we take a random nucleon from the Fermi

12 Indeed, we have $\left|\vec{p}^{\prime}\right|^{2}=\left(\tilde{E}^{\prime}+k_{F}^{2}(r) / 2 M\right)^{2}-M^{2}$.

13 Note that polarization corrections (Eq. (25)) were estimated in isospin symmetric nuclear matter, and thus approximately the RPA term equally contributes to both the proton and neutron $Z^{0}$ absorption channels. 
sea and calculate the initial kinematical variables $\left(P^{\mu}\right.$ and $s$, full four-momentum of the nucleon-nucleon system in the nuclear frame and invariant energy, respectively). Then, a $\cos \theta_{c . m}^{N_{2}}$. is selected, according to the expression given in Eq. (37) below and taken from Ref. [48]. This expression gives us the correct probability given by $d \sigma^{N N} / d \Omega_{c . m}$. plus Pauli blocking restrictions. We take also into account Fermi motion and renormalization effects in the angular dependence. We take them into account by multiplying each event by a weight factor

$$
\xi=\lambda\left(N_{1}, N_{2}\right) \hat{\sigma}^{N_{1} N_{2}} \rho_{N_{2}}
$$

where $1 / \lambda\left(N_{1}, N_{2}\right)$ is the probability by unit of length that one nucleon $N_{1}$ collides with another nucleon $N_{2}$. In average, this factor $\xi$ is equal to one. Explicit formulas are given in the next subsection.

Our method assumes that the nucleons propagate semiclassically in the nucleus. The justification of this hypothesis for reactions induced by real photons is given in Ref. [48], and has also been successfully used in Refs. [46, 50]. In the next section we give some detail on the evaluation of the equivalent $N N$ cross section in the medium.

\section{NN cross sections}

We are using the parameterization of the $N N$ elastic cross section given in the Appendix of Ref. [48]. Since for particles of low momenta, the MC induces large errors, we are not considering collisions of nucleons with kinetic energies below $30 \mathrm{MeV}$. This is to say, we do not follow the path through the nuclear medium of nucleons with kinetic energies below $30 \mathrm{MeV}$, and we just consider that those nucleons get out of the nucleus without suffering further collisions, in which eventually they could change charge or loss some more energy.

On the other hand, the reaction probability will change due to the nuclear medium effects (Fermi motion, Pauli blocking and medium renormalization). Then, according to Ref. [48], the expression for the mean free path $(\lambda)$ of the nucleon is given by

$$
\frac{1}{\lambda\left(N_{1}\right)}=4 \int \frac{d^{3} p_{2}}{(2 \pi)^{3}}\left[\Theta\left(k_{F}^{p}(r)-\left|\vec{p}_{2}\right|\right) \frac{Z}{A} \hat{\sigma}^{N_{1} p}(s)+\Theta\left(k_{F}^{n}(r)-\left|\vec{p}_{2}\right|\right) \frac{(A-Z)}{A} \hat{\sigma}^{N_{1} n}(s)\right] \frac{\left|\vec{p}_{1 \text { lab }}\right|}{\left|\vec{p}_{1}\right|}
$$

with $P^{\mu}$, the full four-momentum of the NN system in the nuclear frame $\left(s=P^{2}=\left(p_{1}+p_{2}\right)^{2}\right), N_{1}$, the incoming nucleon and $N_{2}$, the nucleon in the medium. The factor $\left|\vec{p}_{1 \text { lab }}\right| /\left|\vec{p}_{1}\right|$ is related to the different flux of particles in the nuclear frame and in the nucleon frame $\left(\vec{p}_{1 \text { lab }}\right.$ is the incoming LAB momentum in the $N N$ system and $\vec{p}_{1}$, the momentum in the nuclear system). Furthermore,

$$
\hat{\sigma}^{N_{1} N_{2}}=\int d \Omega_{\mathrm{c} . \mathrm{m} .} \frac{d \sigma^{N_{1} N_{2}}}{d \Omega_{\mathrm{c} . \mathrm{m} .}} \mathbf{C}_{\mathbf{T}}(\mathbf{q}, \rho) \Theta\left(\kappa-\frac{\left|\vec{P} \cdot \vec{p}_{\mathrm{c} . \mathrm{m} .}\right|}{|\vec{P}|\left|\vec{p}_{c . m .}\right|}\right)
$$

where c.m. is the $N N$ center of mass frame and

$$
\kappa=x \Theta(1-|x|)+\frac{x}{|x|} \Theta(|x|-1), \quad x=\frac{P^{0} p_{c . m .}^{0}-\epsilon_{F} \sqrt{s}}{|\vec{P}|\left|\vec{p}_{c . m .}\right|}
$$

where $\vec{p}_{c . m}$. is the nucleon momentum in the c.m. frame, $\epsilon_{F}$ is the Fermi energy $\left(k_{F}^{2} / 2 M\right)$, and the density dependent factor $\mathbf{C}_{\mathbf{T}}$ is defined in Eq. (A9), with $q$ the momentum transfer in the nuclear frame. In these expressions, $\Theta(\kappa-$ $\left.\left|\hat{P} \cdot \hat{p}_{\text {c.m. }}\right|\right)$ takes into account Pauli blocking and $\mathbf{C}_{\mathbf{T}}(\mathbf{q}, \rho)$, the nuclear medium renormalization.

\section{RESULTS}

We compile in Table I the data used for the nuclei studied in this work. As in Ref. [34], nuclear masses and charge densities are taken from Refs. [60] and [61], respectively. The neutron density is taken with the same form as the charge density but properly normalized and with a different radius as suggested by Hartree-Fock calculations [62] and corroborated by pionic atom data [63]. Furthermore, charge densities do not correspond to proton point-like densities because of the finite size of the nucleon. This is taken into account by following the procedure outlined in Section II of Ref. [63].

In the case of $\mathrm{NC}$ driven processes, the minimum energy transfer, $q^{0}$, needed for a proton (neutron) emission reaction corresponds to the proton (neutron) separation energy $Q_{p}\left(Q_{n}\right)$. Our Fermi gas model does not account 


\begin{tabular}{ccccc|cc|cccc}
\hline Nucleus & $R_{p}$ & $R_{n}$ & $a_{p}^{*}$ & $a_{n}^{*}$ & $Q_{p}\left(A_{Z}\right)$ & $Q_{n}\left(A_{Z}\right)$ & $Q_{p}\left(A_{Z+1}\right)$ & $Q_{n}\left(A_{Z-1}\right)$ & $Q$ & $\bar{Q}$ \\
\hline${ }^{16} \mathrm{O}$ & 1.833 & 1.815 & 1.544 & 1.529 & 12.127 & 15.663 & -0.536 & 2.489 & 14.906 & 10.931 \\
${ }^{40} \mathrm{Ar}$ & 3.47 & 3.64 & 0.569 & 0.569 & 12.528 & 9.869 & 7.582 & 5.830 & 0.994 & 7.991 \\
${ }^{40} \mathrm{Ca}$ & 3.51 & 3.43 & 0.563 & 0.563 & 8.328 & 15.641 & 0.539 & 7.799 & 13.809 & 1.822 \\
${ }^{208} \mathrm{~Pb}$ & 6.624 & 6.890 & 0.549 & 0.549 & 8.008 & 9.001 & 3.707 & 3.790 & 2.368 & 5.512 \\
\hline
\end{tabular}

(*) The parameter $a$ is dimensionless for the MHO density form.

TABLE I: Charge $\left(R_{p}, a_{p}\right)$, neutron matter $\left(R_{n}, a_{n}\right)$ density parameters (in fermi units), and $Q_{p(n)}$ proton (neutron) energy separation, $Q-$ and $\bar{Q}-$ values for different nuclei in $\mathrm{MeV}$ units. For the oxygen we use a modified harmonic oscillator (MHO) density, $\rho(r)=\rho_{0}\left(1+a(r / R)^{2}\right) \exp \left(-(r / R)^{2}\right)$, while for the rest of the nuclei, a two-parameter Fermi distribution, $\rho(r)=\rho_{0} /(1+\exp ((r-R) / a))$, was used.

properly for this minimum energy ${ }^{14}$ and we correct it by replacing

$$
q^{0} \rightarrow q^{0}-Q_{p}\left(A_{Z}\right)
$$

for the proton emission reaction and similarly for the neutron one. In the case of CC processes, this minimum excitation energy is $Q+Q_{p}\left(A_{Z+1}\right)\left[\bar{Q}+Q_{n}\left(A_{Z-1}\right)\right]$ for neutrino [antineutrino] induced reactions, with $Q=M\left(A_{Z+1}\right)-M\left(A_{Z}\right)$ $\left[\bar{Q}=M\left(A_{Z-1}\right)-M\left(A_{Z}\right)\right]$.

In what follows, we will present different results for $\mathrm{NC}$ induced inclusive and $\mathrm{NC}$ and $\mathrm{CC}$ nucleon emission reactions at moderate energy transfers to the nucleus, which illustrate the important role played by the different nuclear effects considered in this work: Pauli blocking, RPA and FSI effects, and finally the re-scattering of the outgoing nucleon. For consistency we will always use non-relativistic kinematics to evaluate the contribution of a particle-hole excitation. Relativistic effects were studied in Ref. [34] and found there to be small at the energy regime studied here.

All differential cross sections shown in this section are computed in the LAB frame.

\section{A. Inclusive NC scattering at low energies}

In this section we present results for the inclusive $\mathrm{QE} \mathrm{NC}$ cross sections for both neutrino and antineutrino beams in several nuclei concentrating specially on the nuclear medium effects. The results obtained with the same model for $\mathrm{CC}$ processes can be found in Ref. [34].

In Fig. 3 we show the cross section for the processes $\nu(\bar{\nu})+A \rightarrow \nu(\bar{\nu})+X$ at low and intermediate energies. Above the shown range, pion production could become relevant. We also show for comparison the isospin averaged neutrino (antineutrino) free nucleon cross section.

We find that nuclear effects produce a strong reduction of the cross section. The simple consideration of Pauli blocking is already quite effective even at the higher studied energies. The inclusion of the long range RPA correlations is also quite significant. On the other hand, the magnitude of the reduction is only weakly dependent on the isospin and the atomic mass showing, as expected, a larger effect for heavier nuclei. The size of the effect is similar to the one found in Ref. [34] for CC processes although in this case we also explore the isoscalar pieces of the effective ph-ph interaction responsible of the RPA correlations and the energy thresholds are different.

The addition of FSI (Sect. II C 2) to the calculation containing already Pauli blocking and RPA correlations does not affect practically the results for the totally integrated cross sections.

We show in Fig. 4 the final neutrino energy spectrum for two typical cases. Very similar results are obtained for other nuclei and energies. Although this cross section is not experimentally observable it is useful to show how nuclear effects modify the spectrum and whether they favor some energy transfer. We find that RPA produces a quite smooth reduction that covers all the energy range, although the effects produced by the RPA correlations are weaker when the energy transferred to the nucleus is larger, much the same as it is the case for CC processes. The inclusion of FSI spreads the spectrum allowing for larger energy transfers.

In Fig. 5, we show the ratio of the cross sections induced by neutrino and antineutrinos in oxygen and lead from our full model and compare to the results obtained in the free case: LDT (Eq. (19)). The effect of the nuclear medium in the ratio is quite small although it increases with energy and with the nuclear mass. Similar results were found in Ref. [34] for the CC case.

${ }^{14}$ Indeed, in a Fermi model we need zero excitation energy to emit nucleons. 

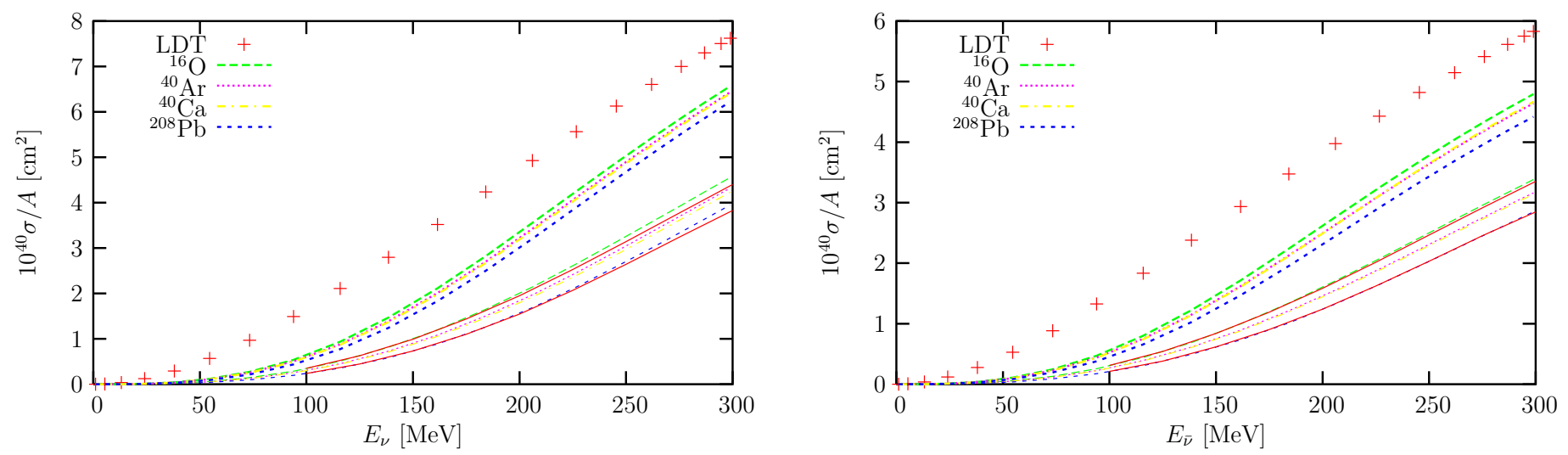

FIG. 3: (color online). Cross section for the $\nu(\bar{\nu})+A \rightarrow \nu(\bar{\nu})+X$ processes as a function of the beam energy. Crosses: $\left(\sigma_{\nu(\bar{\nu})+p \rightarrow \nu(\bar{\nu})+p}+\sigma_{\nu(\bar{\nu})+n \rightarrow \nu(\bar{\nu})+n}\right) / 2$. Intermediate band: Cross sections for several nuclei including Pauli blocking. Lower band: Cross sections for several nuclei with Pauli blocking + RPA correlations. The two solid lines of the lower band correspond to the full model calculation (including also FSI) for oxygen and lead. The left and right panel correspond to neutrino and antineutrino beam respectively.
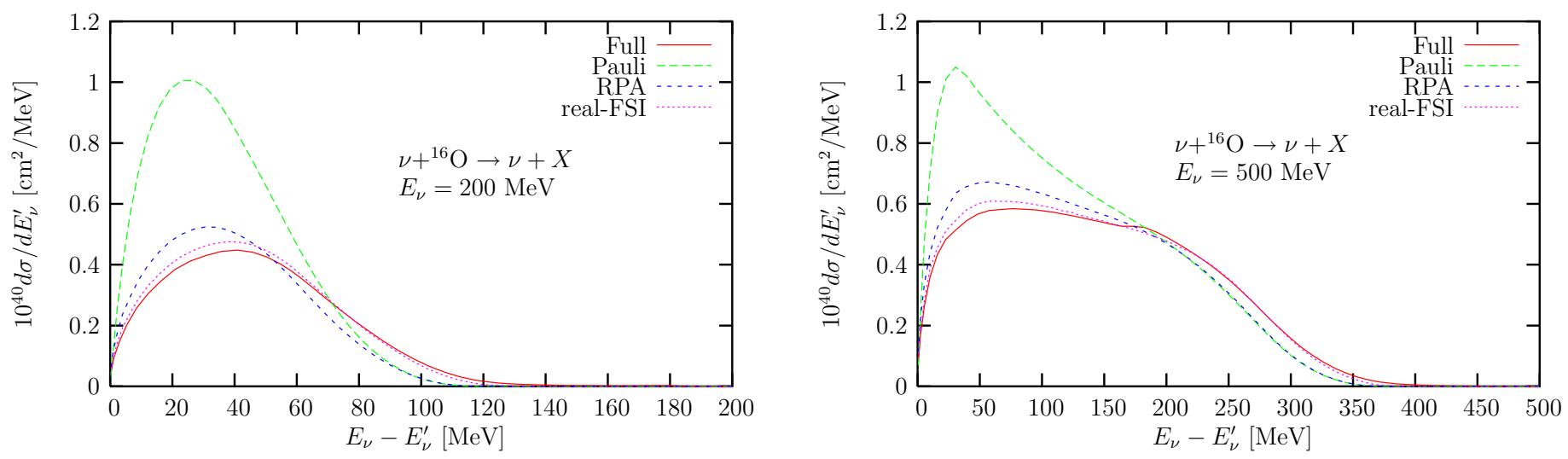

FIG. 4: (color online). NC neutrino $d \sigma / d E_{\nu^{\prime}}$ cross section as a function of the energy transfer and for two different incoming neutrino energies. Predictions from different stages of refinements of the model are shown. Long dashed line: Pauli blocking. Short dashed line: Pauli blocking + RPA. Dotted line: Pauli + RPA + FSI considering only the real part of the nucleon self-energy as explained in the text. Solid line: full model.

\section{B. Inclusive $\left(\nu_{l}, \nu_{l} N\right),\left(\nu_{l}, l^{-} N\right),\left(\bar{\nu}_{l}, \bar{\nu}_{l} N\right)$ and $\left(\bar{\nu}_{l}, l^{+} N\right)$ reactions in nuclei at low energies}

In this section we present results for processes, in which the final nucleons are detected for both neutrino and antineutrino beams in several nuclei. We will also include CC reactions using for this case the model of Ref. [34] in which we have have implemented the nucleons rescattering as described in Sec. III.

As it is illustrated in Fig. 4, the consideration of the dressing of the nucleon propagators (FSI) produces a quenching of the $\mathrm{QE}$ peak respect to the simple ph excitation calculation and a spreading of the strength, or widening of the peak. Most of the effect of the FSI comes from the consideration of the real part of the nucleon selfenergy of Eq. (28) as it is shown in the results obtained taking the limit of vanishing $\operatorname{Im} \Sigma$ (dotted curve in Fig. 4). Thus, we find that the change in the nucleon dispersion relation is more important than the inclusion of the small nucleon width in the medium, related to the quasielastic channels, which will account for $Z^{0}$ absorption by two nucleons. Given the quality of this approximation for the production of the spectra needed to start the MC simulation of the nucleons rescattering, and the considerable reduction in time for its evaluation, it has been used for all the MC results presented in this work. 


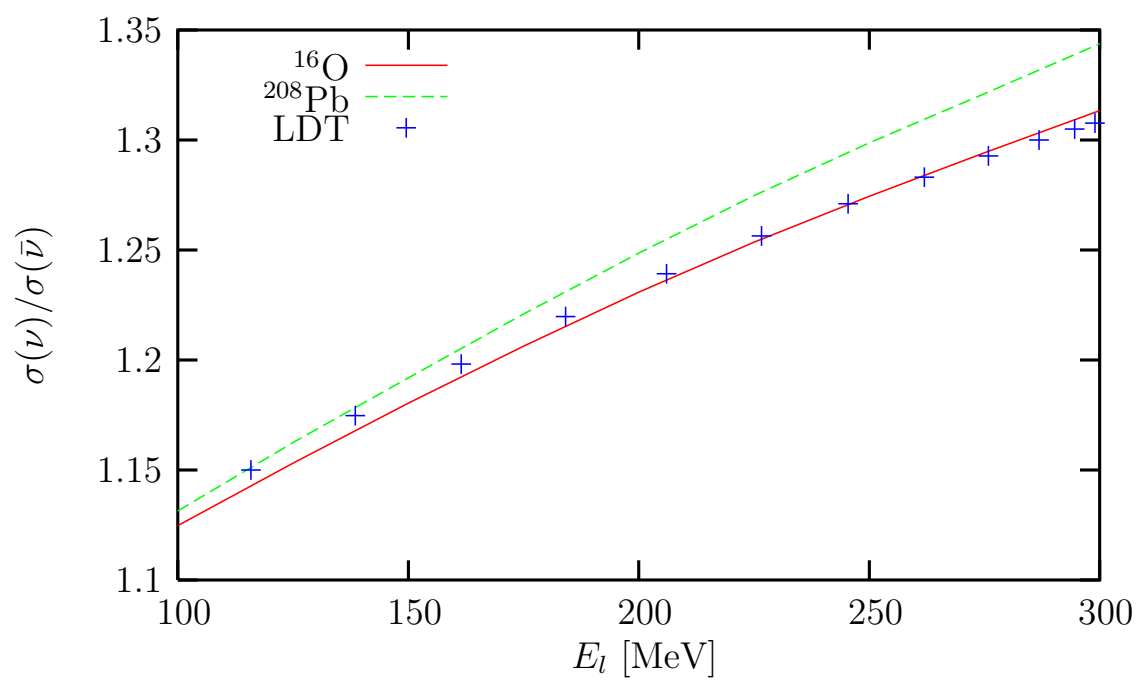

FIG. 5: (color online). $\sigma_{\nu} / \sigma_{\bar{\nu}}$ ratio for the $\nu(\bar{\nu})+A \rightarrow \nu^{\prime}\left(\bar{\nu}^{\prime}\right)+X$ reaction as a function of the energy for several nuclei. LDT means free nucleon results. In all other lines the full model is used.

\section{CC Nucleon spectra}

The nucleon spectra produced by $\mathrm{CC}$ processes induced by muon neutrinos and antineutrinos of $500 \mathrm{MeV}$ are shown in Fig. 6 for argon. Of course neutrinos only interact via CC with neutrons and would emit protons, but these primary protons interact strongly with the medium and collide with other nucleons which are also ejected. As a consequence there is a reduction of the flux of high energy protons but a large number of secondary nucleons, many of them neutrons, of lower energies appear. We should recall that our cascade model does not include the collisions of nucleons with kinetic energies below $30 \mathrm{MeV}$. Thus, the results at those low energies are not meaningful and are shown for illustrative purposes only in Fig.6.

The flux reduction due to the quasielastic NN interaction can be easily accommodated in optical potential calculations. However in those calculations the nucleons that interact are lost when in the physical process they simply come off the nucleus with a different energy and angle, and may be charge, and they must be taken into account.

\section{NC Nucleon spectra}

The energy distributions of nucleons emitted after a NC interaction are shown in Figs. 7 and 8. In Fig. 7, we show the results for ${ }^{40} \mathrm{Ar}$ at two different energies. In both cases we find the large effect of the rescattering of the nucleons. For $500 \mathrm{MeV}$ neutrinos the rescattering of the outgoing nucleon produces a depletion of the higher energies side of the spectrum, but the scattered nucleons clearly enhance the low energies region. For lower neutrino energies, most of the nucleons coming from nucleon nucleon collisions would show up at energies below the $30 \mathrm{MeV}$ cut.

As expected, the rescattering effect is smaller in lighter nuclei as can be seen in Fig. 8 for oxygen. In all cases the final spectra of protons and neutrons are very similar. Our results without rescattering can be compared with other calculations like those of Refs. [22, 32] and other like Refs. [31, 35]. However, in these latter cases, which incorporate the nucleon final state collisions, via the use of optical potentials the main effect of rescattering is to reduce the cross section at all energies instead of displacing the strength towards lower energies as we find.

Also interesting is the very similar spectrum shape obtained in shell model [32] and Fermi gas calculations, as the present one.

Finally, in Fig. 9, we show the effect over the spectrum of the RPA correlations and the consideration of the real part of the nucleon selfenergy (FSI). We find that the larger reduction due to RPA takes place for the lower energy nucleons. A similar situation is obtained for higher energy neutrinos. The inclusion of FSI effects produces an enhancement of the number of high energy protons due to the different nucleon dispersion relation. 

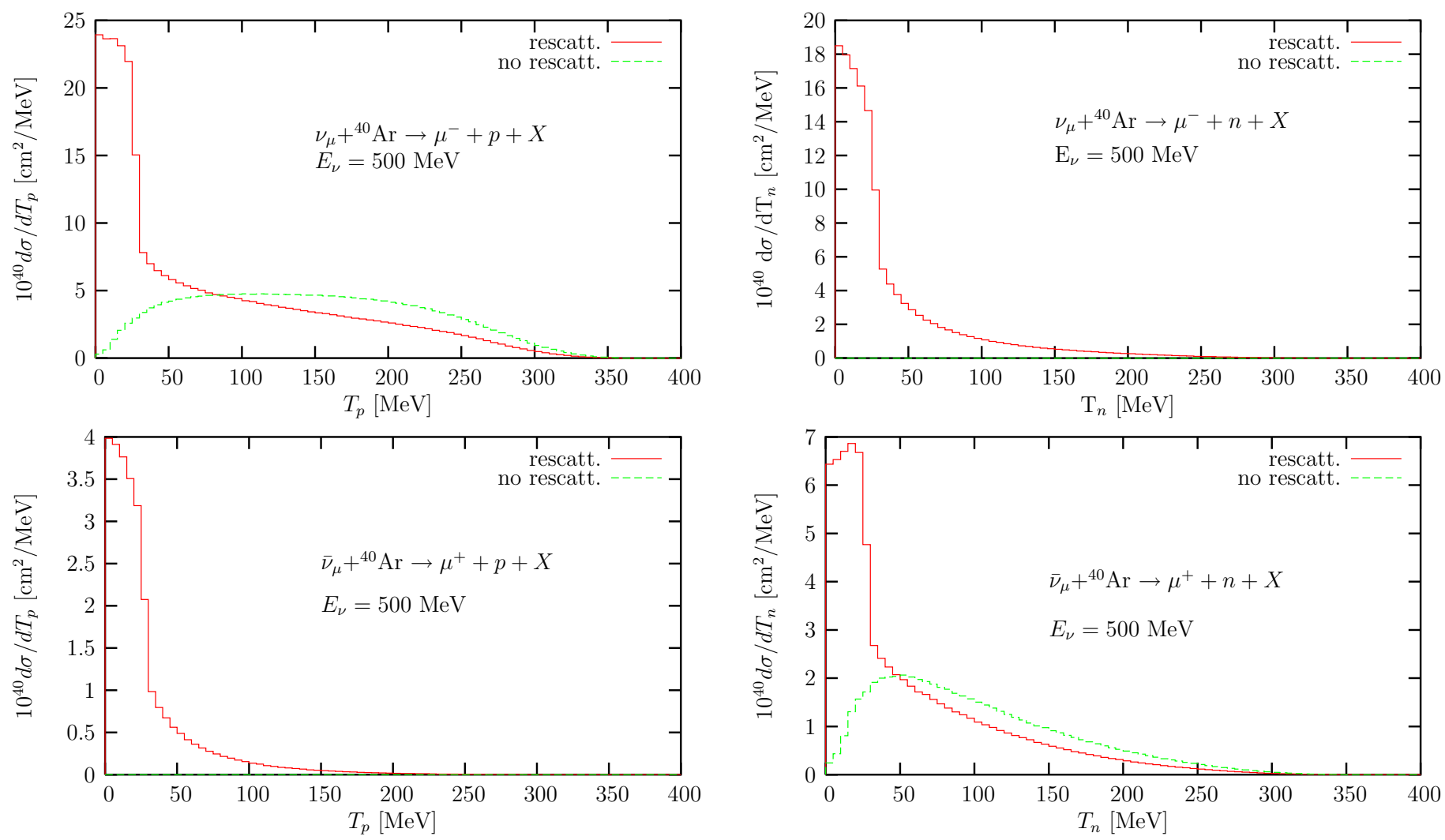

FIG. 6: (color online). Charged current ${ }^{40} A r\left(\nu_{\mu}, \mu^{-}+N\right)$ (upper panels) and ${ }^{40} A r\left(\bar{\nu}_{\mu}, \mu^{+}+N\right)$ (lower panels) cross sections as a function of the kinetic energy of the final nucleon for an incoming neutrino or antineutrino energy of $500 \mathrm{MeV}$. Left and right panels correspond to the emission of protons and neutrons respectively. The dashed histogram shows results without nucleon rescattering and the solid one the full model.

\section{Nucleon angular distributions}

The angular distribution of nucleons is also affected by the rescattering. In Fig. 10, we show the proton and neutron spectra in the $\nu_{\mu}+{ }^{40} A r \rightarrow \mu^{-}+X$ reaction. For comparison we also show the distribution of protons without rescattering. After taking into account the rescattering the proton cross section is less forward peaked. Even flatter is the neutron cross section, because neutrons come only from secondary NN collisions and not from the weak neutrino-nucleon interaction.

For NC reactions (Fig. 11), the situation is more symmetric and the angular distribution is similar for protons and neutrons, as was the case for the energy spectra.

\section{Energy-Angle distributions}

In Fig. 12-15 we show double differential energy-angle cross sections for both charged and neutral currents. Three of the four panels show a common feature with previous calculations (see i.e. Fig. 1 of Ref. [22] and Fig. 5 of Ref. [32]), namely, at forward angles there are two peaks, one of them at low energies, that merge into a single one for larger values of the angle. As it is shown in Ref. [32] the use of the nucleon momentum distributions from shell model wave functions produces less sharp features at forward angles than the Fermi gas calculation of Ref. [22]. However, our results are even softer than those of Ref. [32] although our calculation starts with a local Fermi gas momentum distribution. The reason is clear, the strong effects of rescattering change direction and energy of the nucleons. This can be seen in the panel showing (Fig. 13) the neutron emission for the CC process $\nu_{\mu}+{ }^{16} \mathrm{O} \rightarrow \mu^{-}+n+X$. In this case all neutrons come from nucleon-nucleon collisions and spread over the available phase space without any remarkable feature except the accumulation at low energies. 

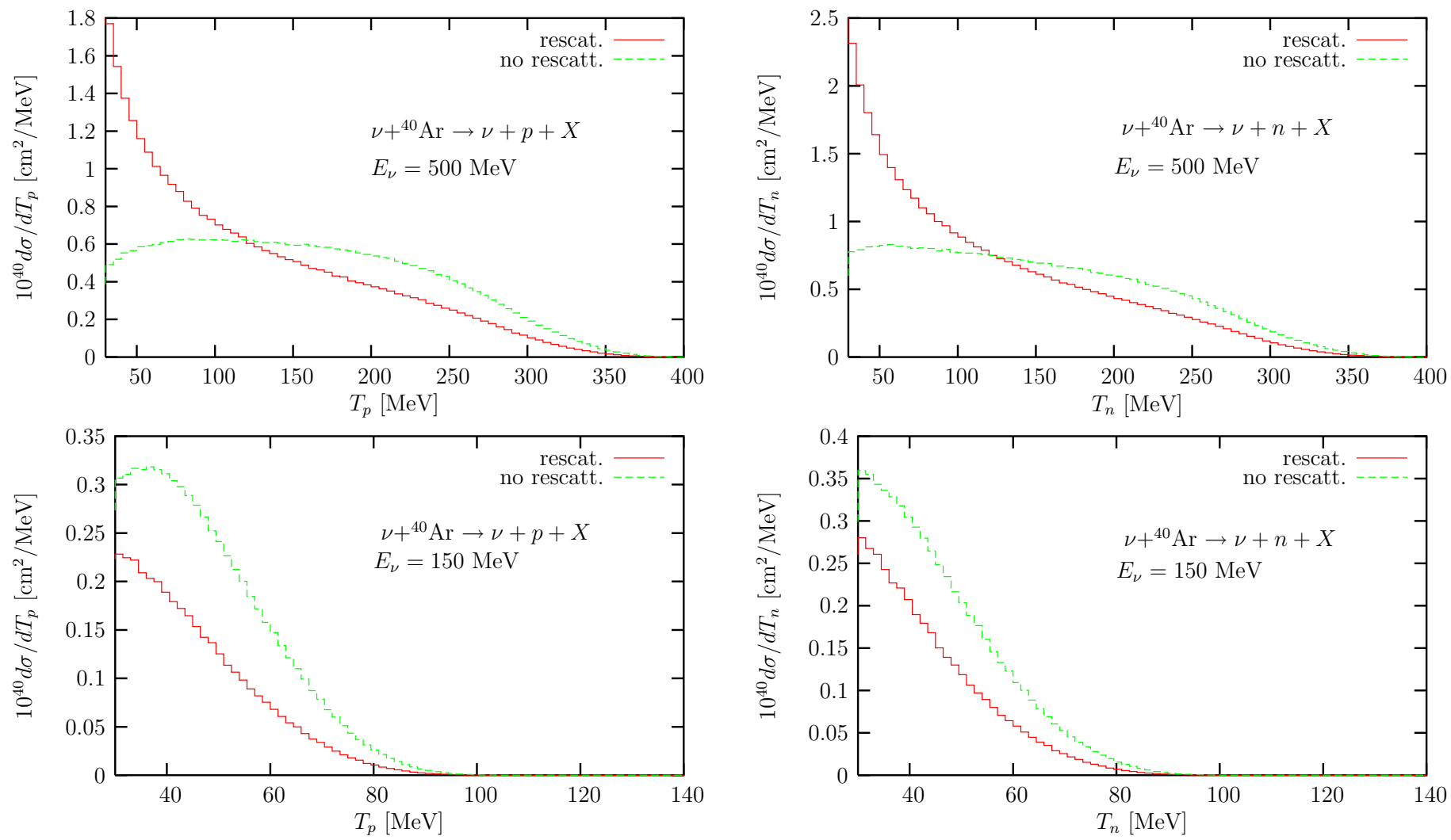

FIG. 7: (color online). Neutral current ${ }^{40} A r(\nu, \nu+N)$ at $500 \mathrm{MeV}$ (upper panels) and $150 \mathrm{MeV}$ (lower panels) cross sections as a function of the kinetic energy of the final nucleon. Left and right panels correspond to the emission of protons and neutrons respectively. The dashed histogram shows results without rescattering and the solid one the full model.

\section{Proton to neutron ratios}

The ratio of proton to neutron QE cross section could be very sensitive to the strange quark axial form factor of the nucleon, and thus to the $g_{A}^{s}$ parameter $[19,20,22,26,32]$. Our results for this ratio in ${ }^{16} \mathrm{O}$ are shown in Fig. 16. We do not consider very low energies where shell model effects could be more important and our MC simulation is unreliable. We find similar results for light nuclei and low energies as in Ref. [20] where RPA correlations were taken into account or in Ref. [22]. Our model includes as the main additional ingredient the rescattering of the nucleons via a $\mathrm{MC}$ simulation. However also this rescattering produces minor changes for light nuclei, because of the smaller average density, and for low energies because most secondary nucleons are below our $30 \mathrm{MeV}$ cut. However, the sensitivity to the collisions of the final nucleons is larger for both heavier nuclei and for larger energies of the neutrinos as shown in Fig. 17 where it is clear that one sees the importance of the secondary nucleons at the low energies side of the spectrum.

\section{CONCLUSIONS}

We have studied the QE contribution to the inclusive $\left(\nu_{l}, \nu_{l} N\right),\left(\nu_{l}, l^{-} N\right),\left(\bar{\nu}_{l}, \bar{\nu}_{l} N\right)$ and $\left(\bar{\nu}_{l}, l^{+} N\right)$ reactions in nuclei using a MC simulation method to account for the collisions of the ejected nucleons during their way out of the nucleus. As input, we have used the reaction probability from the microscopical many body framework developed in Ref. [34] for $\mathrm{CC}$ induced reactions, while for $\mathrm{NC}$ we use results from a natural extension, performed also in this work, of the model described in that reference. Limitations of the DWIA models have been discussed. In particular those models cannot properly describe individual inclusive neutron and proton spectra and for the ratio of proton $(\nu, p)$ to neutron $(\nu, n)$ yields, the sensitivity to the collisions of the final nucleons might become important for both medium and heavy nuclei and for energies of the neutrinos larger than $150 \mathrm{MeV}$, as shown in Fig. 17. 

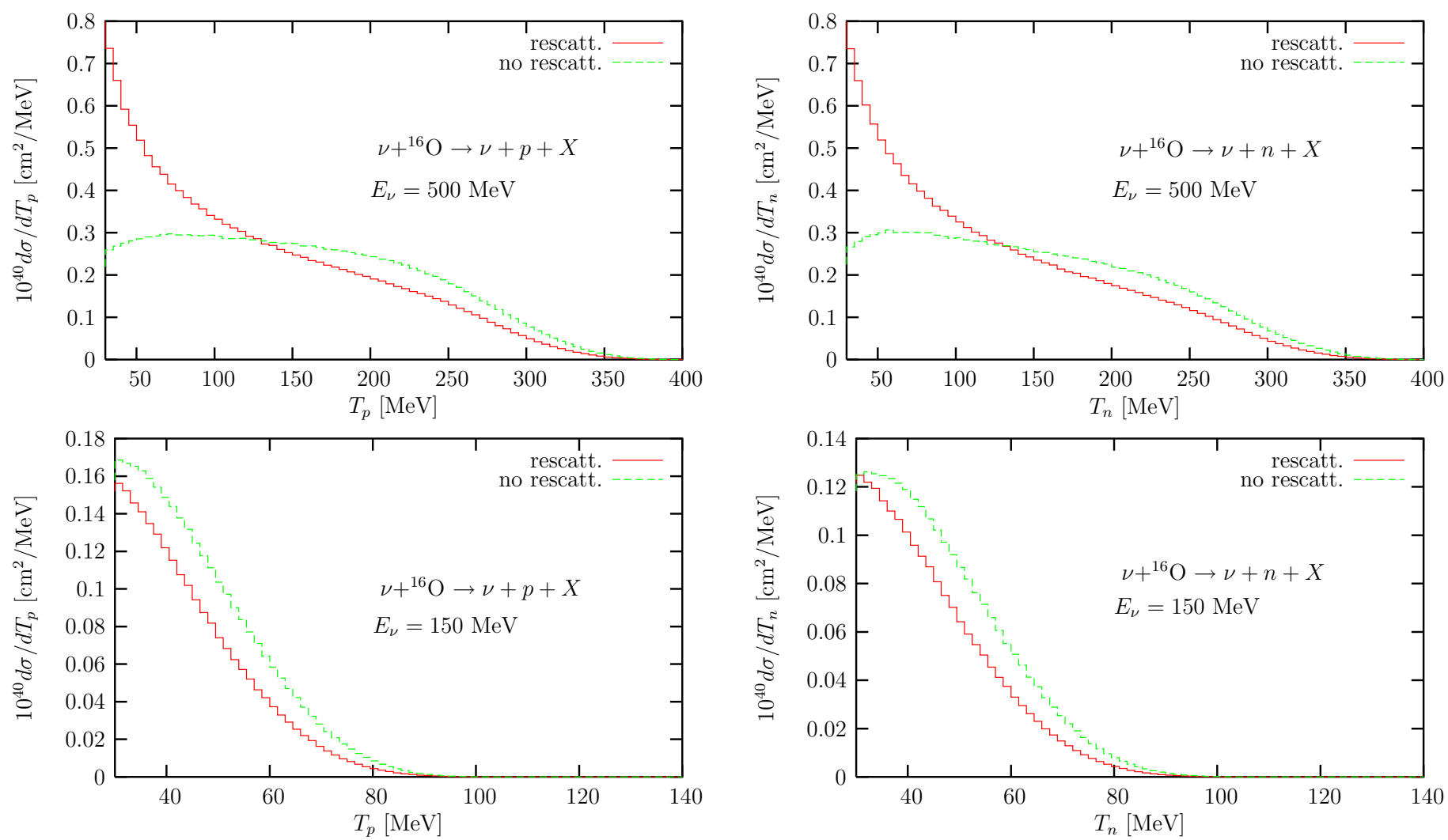

FIG. 8: (color online). Same as Fig.7 for oxygen.

\section{Acknowledgments}

J.N. warmly thanks to E. Oset for various stimulating discussions and communications. This work was supported by DGI and FEDER funds, contracts BFM2002-03218 and BFM2003-00856, by the EU Integrated Infrastructure Initiative Hadron Physics Project contract RII3-CT-2004-506078 and by the Junta de Andalucía.

\section{APPENDIX A: NC NUCLEON TENSOR}

\section{Impulse Approximation}

Taking into account that in Eq. (17) both the particle and the hole nucleons are on the mass shell $\left(p^{2}=(p+q)^{2}=\right.$ $M^{2}, 2 p \cdot q+q^{2}=0$ ), one finds

$$
A_{N}^{\mu \nu}(p, q)=a_{1}^{N} g^{\mu \nu}+a_{2}^{N}\left(p^{\mu} p^{\nu}+\frac{p^{\mu} q^{\nu}+p^{\nu} q^{\mu}}{2}\right)+\mathrm{i} a_{3}^{N} \epsilon^{\mu \nu \alpha \beta} p_{\alpha} q_{\beta}+a_{4}^{N} q^{\mu} q^{\nu}
$$

with, omitting the obvious subindex $N=n$ or $p$,

$$
\begin{aligned}
& a_{1}\left(q^{2}\right)=8 q^{2}\left\{\left(F_{1}^{Z}+\mu_{Z} F_{2}^{Z}\right)^{2}+\left(G_{A}^{Z}\right)^{2}\left(\frac{1}{4}-\frac{M^{2}}{q^{2}}\right)\right\} \\
& a_{2}\left(q^{2}\right)=32\left(F_{1}^{Z}\right)^{2}-8\left(\mu_{Z} F_{2}^{Z}\right)^{2} \frac{q^{2}}{M^{2}}+8\left(G_{A}^{Z}\right)^{2} \\
& a_{3}\left(q^{2}\right)=16 G_{A}^{Z}\left(F_{1}^{Z}+\mu_{Z} F_{2}^{Z}\right) \\
& a_{4}\left(q^{2}\right)=-\frac{8 q^{2}}{M^{2}}\left(\mu_{Z} F_{2}^{Z}\right)^{2}\left(\frac{M^{2}}{q^{2}}+\frac{1}{4}\right)-16 F_{1}^{Z} \mu_{Z} F_{2}^{Z}
\end{aligned}
$$




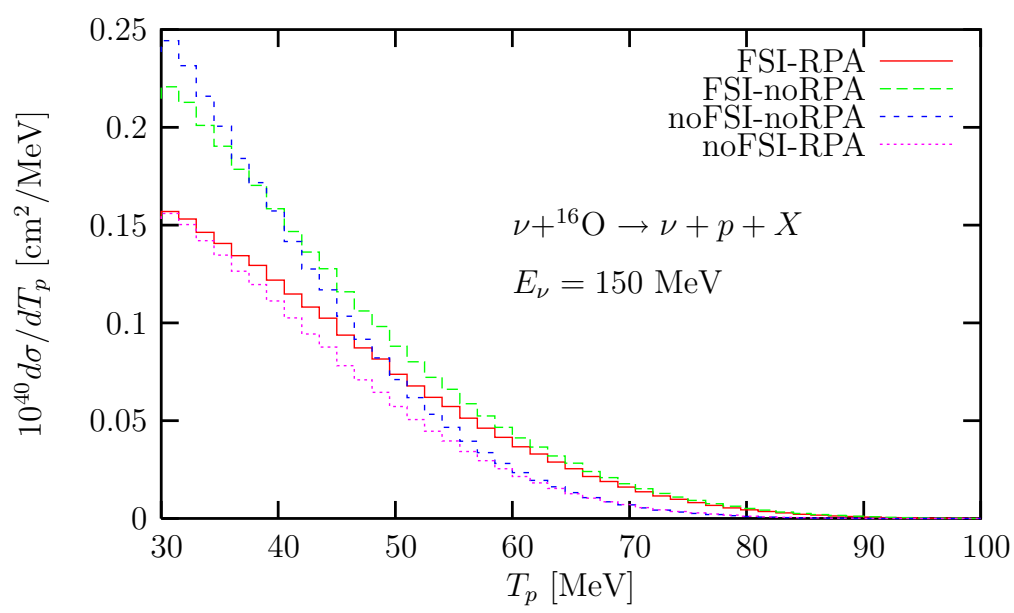

FIG. 9: (color online). $d \sigma / d T_{p}^{\prime}$ cross section for oxygen at $E_{\nu}=150 \mathrm{MeV}$ as a function of the kinetic energy of the final proton for different nuclear models, including or not RPA and FSI effects. In all cases a MC simulation is performed to compute the rescattering of the outgoing nucleons.

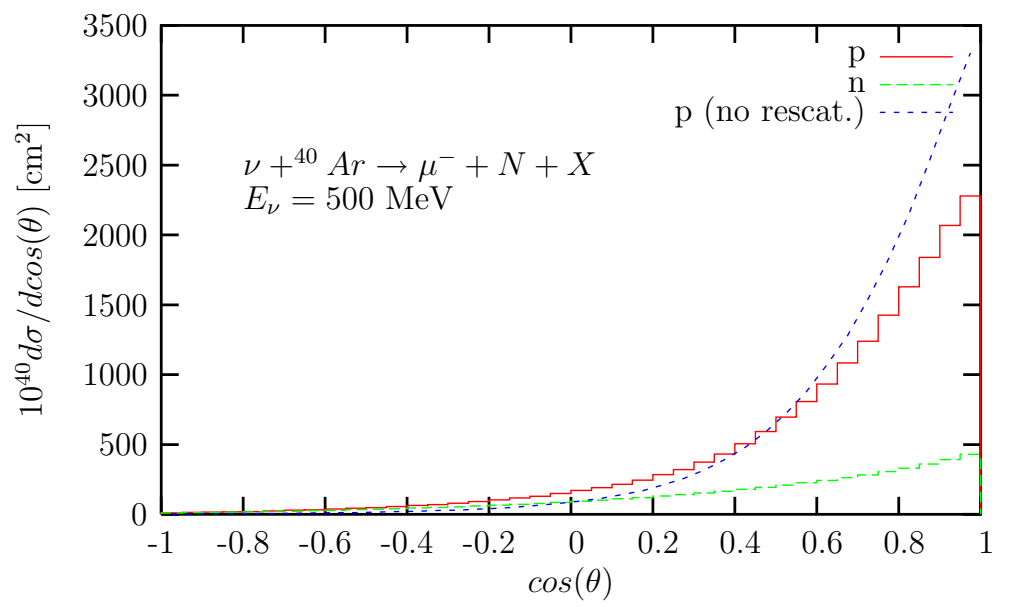

FIG. 10: (color online). Nucleon angular distribution for the ${ }^{40} A r\left(\nu_{\mu}, \mu^{-}+N\right)$ reaction. The dashed line shows the proton results without rescattering and the solid (dotted) histogram stands for proton (neutron) results from our full model. In all cases, the contribution of low energy nucleons (below $30 \mathrm{MeV}$ ) is not included.

The cross section for the process $\nu_{l}+N \rightarrow \nu_{l}+N$ is given by

$$
\sigma_{\nu \nu}=\frac{G^{2}}{32 \pi\left(s-M^{2}\right)^{2}} \int_{-\left(s-M^{2}\right)^{2} / s}^{0} d q^{2}\left(q^{2}\left\{a_{1}+\frac{s}{2} a_{2}-\frac{q^{2}}{2} a_{3}\right\}+\left(s-M^{2}\right)\left\{\frac{s-M^{2}}{2} a_{2}-q^{2} a_{3}\right\}\right)
$$

where $s=(2|\vec{k}|+M) M$ is the Mandelstam variable $(|\vec{k}|$ is incoming neutrino energy in the LAB frame). The variable $q^{2}$ is related to the outgoing neutrino LAB polar angle $\left(\theta^{\prime}\right)$ by $q^{2}=\left(k-k^{\prime}\right)^{2}=-2\left|\vec{k} \| \vec{k}^{\prime}\right|\left(1-\cos \theta^{\prime}\right)$.

The cross section for the process $\bar{\nu}_{l}+N \rightarrow \bar{\nu}_{l}+N$ is obtained by replacing $a_{3}$ by $-a_{3}$. 


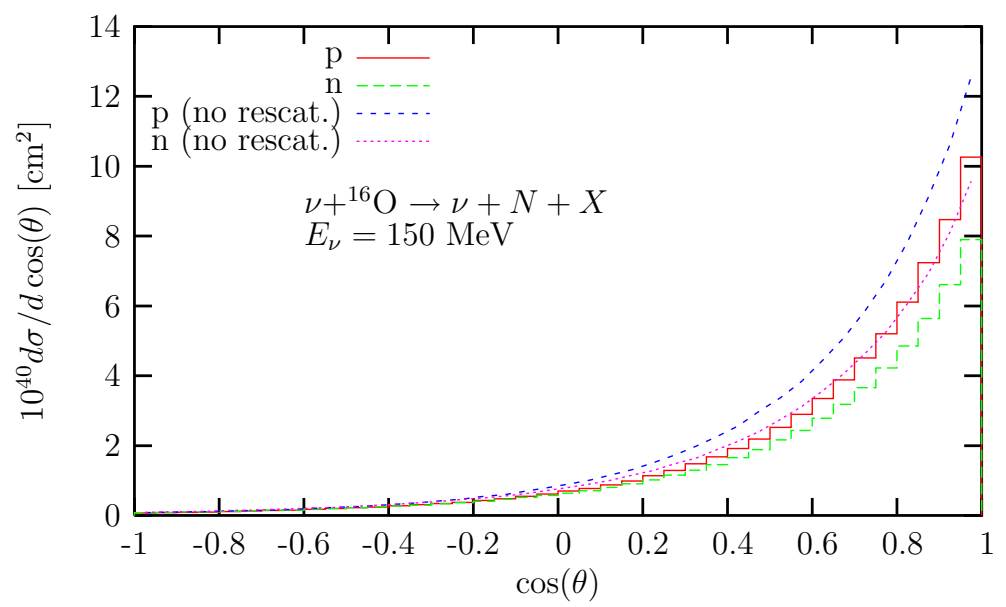

FIG. 11: (color online). Proton and neutron angular distribution for the ${ }^{16} O\left(\nu, \nu^{\prime}+N\right)$ reaction. In all cases, the contribution of low energy nucleons (below $30 \mathrm{MeV}$ ) is not included.

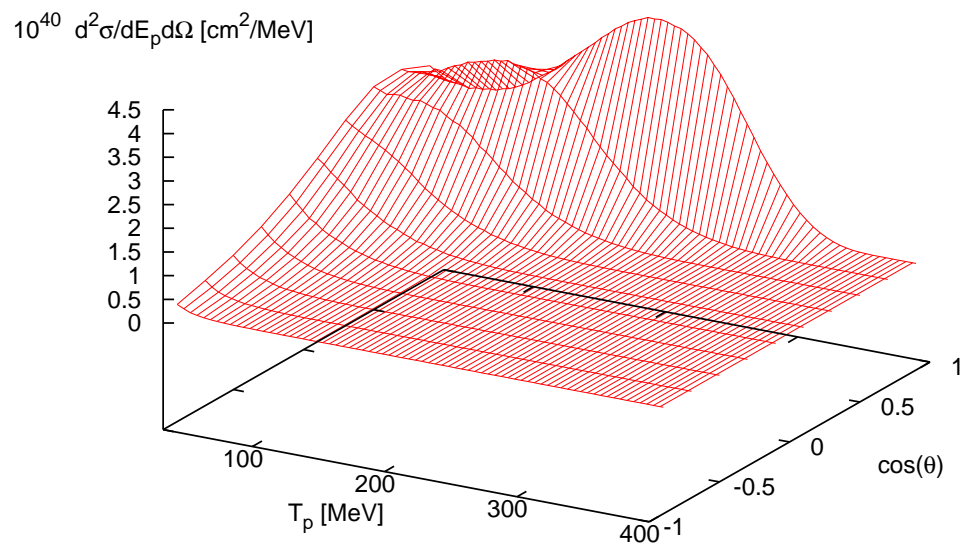

FIG. 12: (color online). Double differential cross section $d^{2} \sigma / d \Omega d E$ as a function of the outgoing kinetic nucleon energy and scattering angle for the reaction $\nu_{\mu}+{ }^{16} \mathrm{O} \rightarrow \mu^{-}+p+X$ and an incoming neutrino energy of $500 \mathrm{MeV}$.

\section{RPA Corrections}

Taking $\vec{q}$ in the $z$ direction and after performing the RPA sum of Fig. 2, we find, neglecting ${ }^{15}$ corrections of order $\mathcal{O}\left(k_{F} \vec{p}^{2} / M^{2}, k_{F} \vec{p}^{2} / M^{2}, k_{F} q^{0} / M\right)$

$$
\begin{aligned}
\frac{\delta A_{\mathrm{RPA}}^{00}}{2 M^{2}} & =8\left(\frac{E(\vec{p})}{M}\right)^{2}\left\{\left(\mathbf{C}_{\mathbf{N}}-1\right)\left[\left(F_{1}^{Z}\right)^{p}-\left(F_{1}^{Z}\right)^{n}\right]^{2}+\left(\mathbf{D}_{\mathbf{N}}-1\right)\left[\left(F_{1}^{Z}\right)^{p}+\left(F_{1}^{Z}\right)^{n}\right]^{2}\right\} \\
& -4 \frac{\vec{q}^{2}}{M^{2}}\left\{\left(\mathbf{C}_{\mathbf{N}}-1\right)\left[\left(F_{1}^{Z}\right)^{p}-\left(F_{1}^{Z}\right)^{n}\right]\left[\left(\mu_{Z} F_{2}^{Z}\right)^{p}-\left(\mu_{Z} F_{2}^{Z}\right)^{n}\right]\right. \\
& \left.+\left(\mathbf{D}_{\mathbf{N}}-1\right)\left[\left(F_{1}^{Z}\right)^{p}+\left(F_{1}^{Z}\right)^{n}\right]\left[\left(\mu_{Z} F_{2}^{Z}\right)^{p}+\left(\mu_{Z} F_{2}^{Z}\right)^{n}\right]\right\} \\
\frac{\delta A_{\mathrm{RPA}}^{0 z}}{2 M^{2}} & =\left(\frac{E(\vec{p})}{M} \frac{2 p_{z}+|\vec{q}|}{M}\right)\left\{4\left(\mathbf{C}_{\mathbf{N}}-1\right)\left[\left(F_{1}^{Z}\right)^{p}-\left(F_{1}^{Z}\right)^{n}\right]^{2}+4\left(\mathbf{D}_{\mathbf{N}}-1\right)\left[\left(F_{1}^{Z}\right)^{p}+\left(F_{1}^{Z}\right)^{n}\right]^{2}\right.
\end{aligned}
$$

15 Note that $q^{0} / M$ is of the order $|\vec{q}|^{2} / M^{2}$ and as mentioned in Sect. II C, we have considered $\mu_{Z} F_{2}^{Z}|\vec{q}| / M$ of order $\mathcal{O}(0)$. 


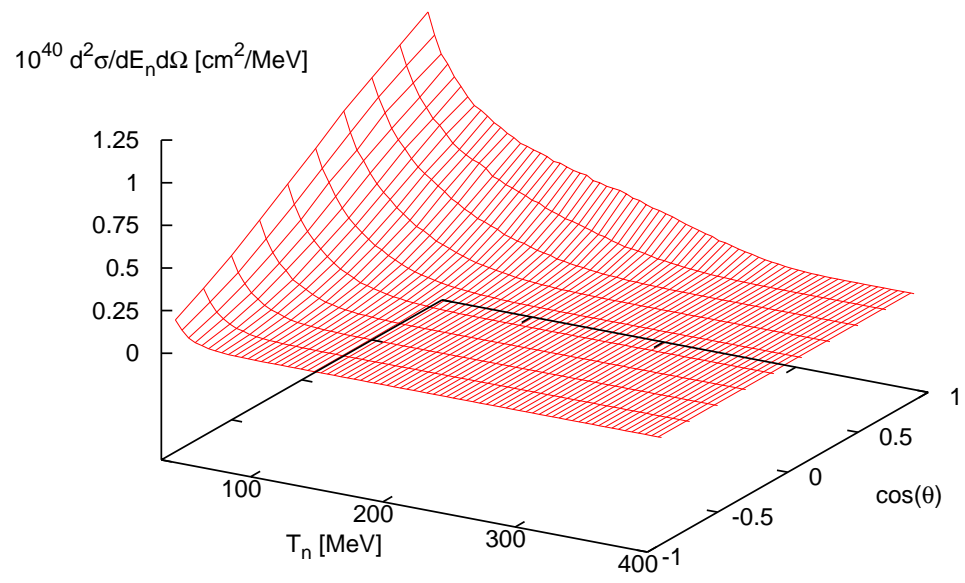

FIG. 13: (color online). Same as Fig.12 for $\nu_{\mu}+{ }^{16} \mathrm{O} \rightarrow \mu^{-}+n+X$

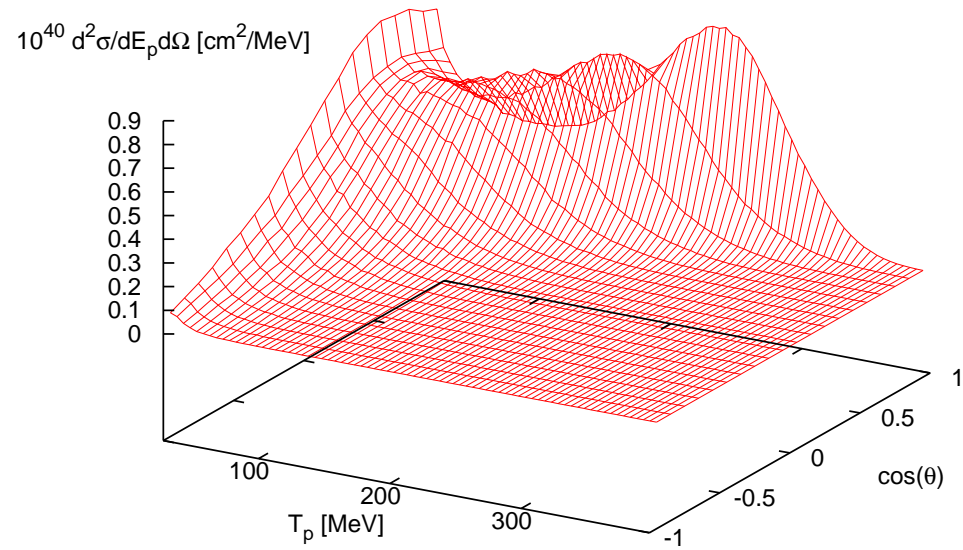

FIG. 14: (color online). Same as Fig.12 for $\nu+{ }^{16} \mathrm{O} \rightarrow \nu+p+X$. Incoming neutrino energy $500 \mathrm{MeV}$

$$
\begin{aligned}
& \left.+\left(\mathbf{C}_{\mathbf{L}}-1\right)\left[\left(G_{A}^{Z}\right)^{p}-\left(G_{A}^{Z}\right)^{n}\right]^{2}+\left(\mathbf{E}_{\mathbf{N}}-1\right)\left[\left(G_{A}^{Z}\right)^{p}+\left(G_{A}^{Z}\right)^{n}\right]^{2}\right\} \\
\frac{\delta A_{\mathrm{RPA}}^{z z}}{2 M^{2}} & =2\left(\mathbf{C}_{\mathbf{L}}-1\right)\left[\left(G_{A}^{Z}\right)^{p}-\left(G_{A}^{Z}\right)^{n}\right]^{2}+2\left(\mathbf{E}_{\mathbf{N}}-1\right)\left[\left(G_{A}^{Z}\right)^{p}+\left(G_{A}^{Z}\right)^{n}\right]^{2} \\
\frac{\delta A_{\mathrm{RPA}}^{x x}}{2 M^{2}} & \left.\left.\left.=-2 \frac{q^{2}}{M^{2}}\left\{\left(\mathbf{C}_{\mathbf{T}}-1\right)\left[\left(\mu_{Z} F_{2}^{Z}\right)^{p}-\left(\mu_{Z} F_{2}^{Z}\right)\right)^{n}\right]^{2}+\left(\mathbf{E}_{\mathbf{N}}-1\right)\left[\left(\mu_{Z} F_{2}^{Z}\right)\right)^{p}+\left(\mu_{Z} F_{2}^{Z}\right)\right)^{n}\right]^{2}\right\} \\
& -4 \frac{q^{2}}{M^{2}}\left\{\left(\mathbf{C}_{\mathbf{T}}-1\right)\left[\left(F_{1}^{Z}\right)^{p}-\left(F_{1}^{Z}\right)^{n}\right]\left[\left(\mu_{Z} F_{2}^{Z}\right)^{p}-\left(\mu_{Z} F_{2}^{Z}\right)^{n}\right]\right. \\
& \left.+\left(\mathbf{E}_{\mathbf{N}}-1\right)\left[\left(F_{1}^{Z}\right)^{p}+\left(F_{1}^{Z}\right)^{n}\right]\left[\left(\mu_{Z} F_{2}^{Z}\right)^{p}+\left(\mu_{Z} F_{2}^{Z}\right)^{n}\right]\right\} \\
& +2\left(\mathbf{C}_{\mathbf{T}}-1\right)\left[\left(G_{A}^{Z}\right)^{p}-\left(G_{A}^{Z}\right)^{n}\right]^{2}+2\left(\mathbf{E}_{\mathbf{N}}-1\right)\left[\left(G_{A}^{Z}\right)^{p}+\left(G_{A}^{Z}\right)^{n}\right]^{2} \\
\frac{\delta A_{\mathrm{RPA}}^{x y}}{2 M^{2}} & =-4 \mathrm{i} \frac{|\vec{q}| E(\vec{p})}{M^{2}}\left\{\left(\mathbf{C}_{\mathbf{T}}-1\right)\left[\left(G_{A}^{Z}\right)^{p}-\left(G_{A}^{Z}\right)^{n}\right]\left[\left(F_{1}^{Z}+\mu_{Z} F_{2}^{Z}\right)^{p}-\left(F_{1}^{Z}+\mu_{Z} F_{2}^{Z}\right)^{n}\right]\right. \\
& \left.+\left(\mathbf{E}_{\mathbf{N}}-1\right)\left[\left(G_{A}^{Z}\right)^{p}+\left(G_{A}^{Z}\right)^{n}\right]\left[\left(F_{1}^{Z}+\mu_{Z} F_{2}^{Z}\right)^{p}+\left(F_{1}^{Z}+\mu_{Z} F_{2}^{Z}\right)^{n}\right]\right\}
\end{aligned}
$$

with the polarization coefficients defined as

$$
\mathbf{C}_{\mathbf{N}}(\rho)=\frac{1}{\left|1-c_{0} f_{0}^{\prime}(\rho) U_{N}\left(q, k_{F}\right)\right|^{2}}, \quad \mathbf{C}_{\mathbf{T}}(\rho)=\frac{1}{\left|1-U\left(q, k_{F}\right) V_{t}(q)\right|^{2}} \quad \mathbf{C}_{\mathbf{L}}(\rho)=\frac{1}{\left|1-U\left(q, k_{F}\right) V_{l}(q)\right|^{2}}
$$




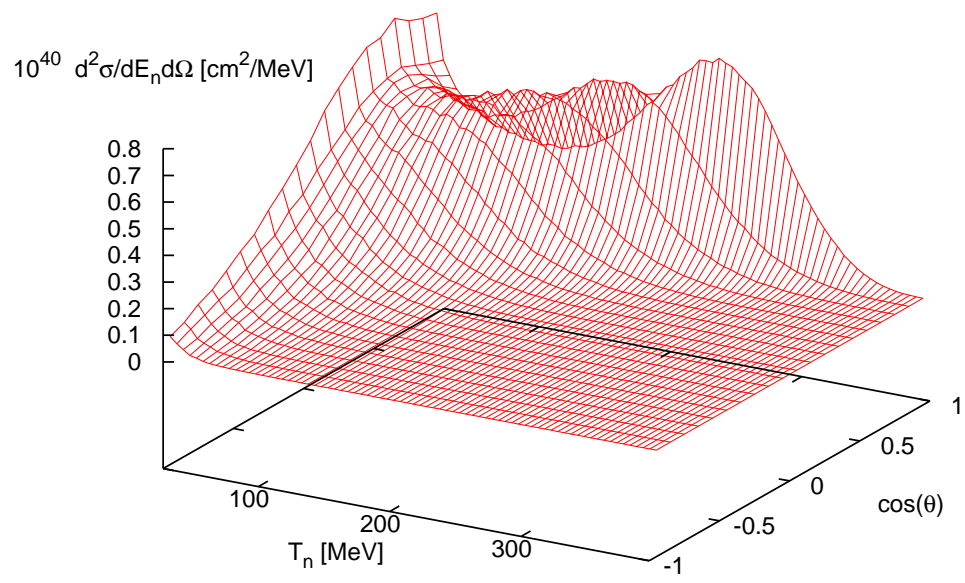

FIG. 15: (color online). Same as Fig.12 for $\nu+{ }^{16} \mathrm{O} \rightarrow \nu+n+X$. Incoming neutrino energy $500 \mathrm{MeV}$

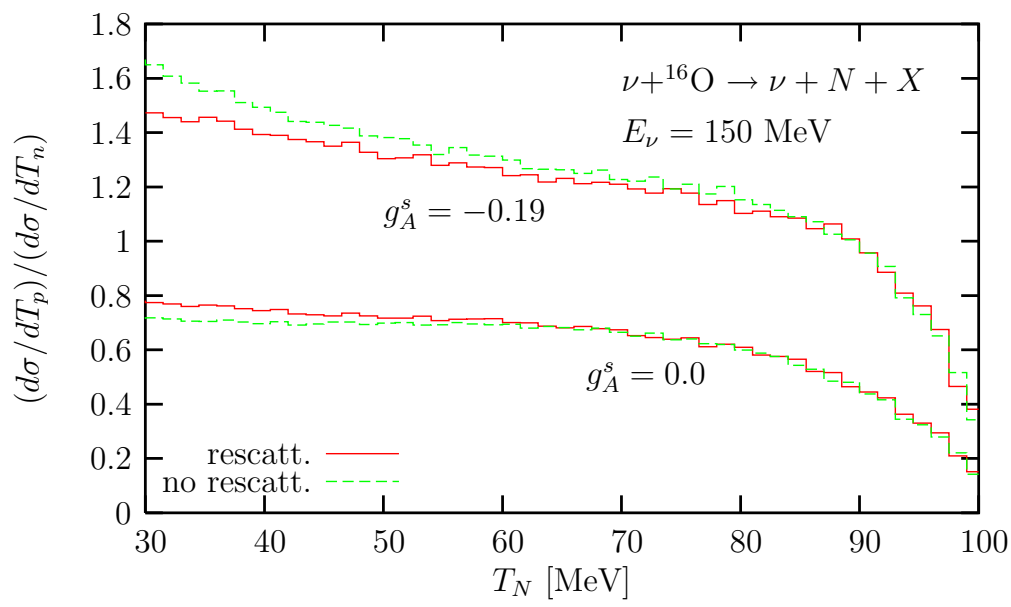

FIG. 16: (color online). Ratio of $d \sigma / d T$ for protons over that for neutrons for $E_{\nu}=150 \mathrm{MeV}$ in the reaction $\nu+{ }^{16} O \rightarrow \nu^{\prime}+N+X$ as a function of the nucleon kinetic energy. Dashed histogram: without nucleon rescattering. Solid histogram: full model.

$$
\mathbf{D}_{\mathbf{N}}(\rho)=\frac{1}{\left|1-c_{0} f_{0}(\rho) U_{N}\left(q, k_{F}\right)\right|^{2}}, \quad \mathbf{E}_{\mathbf{N}}(\rho)=\frac{1}{\left|1-c_{0} g_{0}(\rho) U_{N}\left(q, k_{F}\right)\right|^{2}}
$$

[1] Super-Kamiokande Collaboration, Y. Fukuda et al., Phys. Rev. Lett. 81 (1998) 1562; The K2K Collaboration, M. Ahn et al., Phys. Rev. Lett. 90 (2003) 041801.

[2] See different talks at ' $\nu$-RCCN International Workshop', RCNN, Kashiwa, Japan, December 2004 (http://www-rccn.icrr.u-tokyo.ac.jp/rccnws04).

[3] O. Benhar, N. Farina, H. Nakamura, M. Sakuda and R. Seki, Phys. Rev. D 72 (2005) 053005

[4] EMC Collaboration, J. Ashan et al., Nucl. Phys. B328 (1989) 1.

[5] L. A. Ahrens et al., Phys. Rev. D35 (1987) 785.

[6] J.R. Ellis and R. L. Jaffe, Phys. Rev. D9 (1974) 1444.

[7] FINeSSe Collaboration, L. Bugel et al., "A proposal for a near detector experiment on the booster neutrino beamline: FINeSSE: Fermilab Intense Neutrino Scattering Scintillator Experiment", hep-ex/0402007.

[8] S. F. Pate, Eur. Phys. J. A 24S2 (2005) 67 [arXiv:nucl-ex/0502014].

[9] A. Botrugno and G. Co', Nucl. Phys. A761 (2005) 200.

[10] E. Kolbe, K. Langanke, F.K. Thielemann and P. Vogel, Phys. Rev. C52 (1995) 3437; E. Kolbe, K. Langanke and S. Krewald, Phys. Rev. C49 (1994) 1122; E. Kolbe, K. Langanke and P. Vogel, Nucl. Phys. A613 (1997) 382; ibidem Nucl. Phys. A652 (1999) 91. 

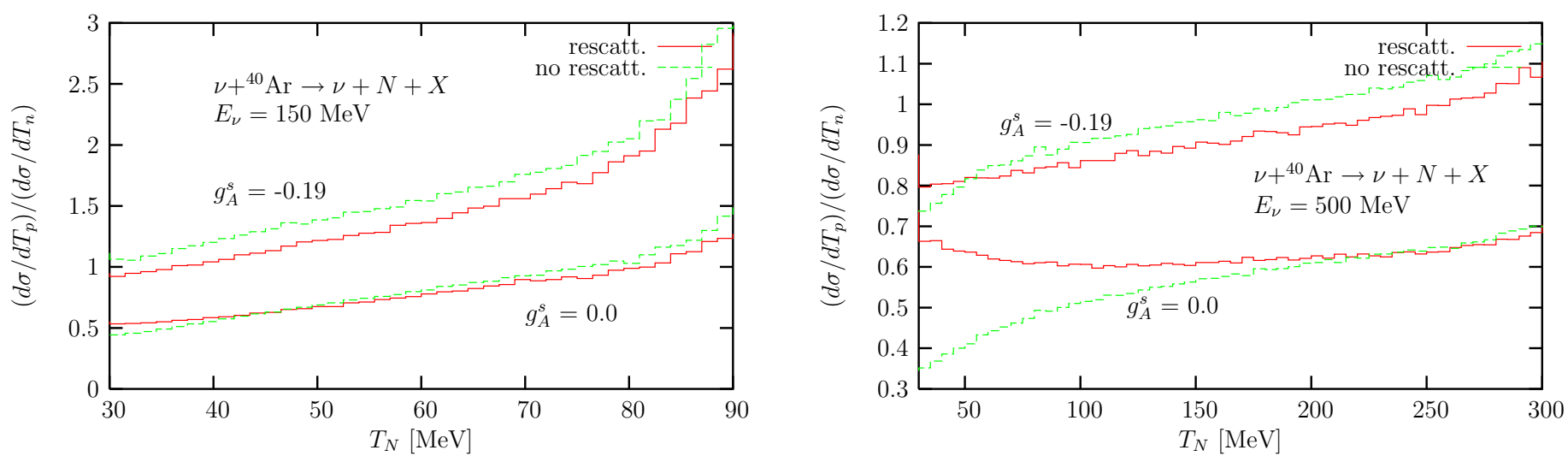

FIG. 17: (color online). Ratio of $d \sigma / d E$ for protons over that for neutrons for $E_{\nu}=150 \mathrm{MeV}$ and $E_{\nu}=500 \mathrm{MeV}$ in the reaction $\nu+{ }^{40} A r \rightarrow \nu^{\prime}+N+X$ as a function of the nucleon kinetic energy. Dashed histogram: without nucleon rescattering. Solid histogram: full model.

[11] N. Auerbach, N. Van Giai and O.K. Vorov, Phys. Rev. C56 (1997) R2368; N. Auerbach, C. Volpe, G. Colo, T. Suzuki and N. Van Giai, Nucl. Phys. A687 (2001) 289.

[12] N. Jachowicz, S. Rombouts, K. Heyde, and J. Ryckebusch, Phys. Rev. C59 (1999) 3246; N. Jachowicz, K. Heyde, J. Ryckebusch and S. Rombouts, Phys. Rev. C65 (2002) 025501.

[13] A.C. Hayes and I.S. Towner, Phys. Rev. C61 (2000) 044603.

[14] N. Auerbach, B.A. Brown, Phys. Rev.C65 (2002) 024322.

[15] E. Kolbe, K. Langanke, G. Martínez-Pinedo and P.Vogel, J. Phys. G29 (2003) 2569.

[16] L. Alvarez-Ruso, S. K. Singh and M. J. Vicente Vacas, Phys. Rev. C 59 (1999) 3386; Phys. Rev. C 57 (1998) 2693.

[17] S. K. Singh, M. J. Vicente-Vacas and E. Oset, Phys. Lett. B 416 (1998) 23 [Erratum-ibid. B 423 (1998) 428].

[18] O. Lalakulich and E. A. Paschos, Phys. Rev. D 71 (2005) 074003.

[19] G. T. Garvey, S. Krewald, E. Kolbe and K. Langanke, Phys. Lett. B 289 (1992) 249.

[20] G. Garvey, E. Kolbe, K. Langanke and S. Krewald, Phys. Rev. C 48 (1993) 1919.

[21] S.K. Singh and E. Oset, Nucl. Phys. A542 (1992) 587; ibidem Phys. Rev. C48 (1993) 1246; T.S. Kosmas and E. Oset, Phys. Rev. 53 (1996) 1409; S.K. Singh, N.C. Mukhopadhyay and E. Oset, Phys. Rev. C57 (1998) 2687.

[22] C. J. Horowitz, H. C. Kim, D. P. Murdock and S. Pollock, Phys. Rev. C 48 (1993) 3078.

[23] H. C. Kim, J. Piekarewicz and C. J. Horowitz, Phys. Rev. C 51 (1995) 2739.

[24] Y. Umino, and J.M. Udias, Phys. Rev. C52 (1995) 3399; Y. Umino, J.M. Udias and P.J. Mulders, Phys. Rev. Lett. 74 (1995) 4993.

[25] S.L. Mintz and M. Pourkaviani, Nucl. Phys. A594 (1995) 346.

[26] W.M. Alberico, M.B. Barbaro, S.M. Bilenky, J.A. Caballero, C. Giunti, C. Maieron, E. Moya de Guerra, and J.M. Udías, Nucl. Phys. A623 (1997) 471; Phys. Lett. B438 (1998) 9; Nucl. Phys. A651 (1999) 277.

[27] C. Volpe, N. Auerbach, G. Colo, T. Suzuki and N. Van Giai, Phys. Rev. C62 (2000) 015501

[28] C. Bleve, G. Co', I. De Mitri, P. Bernardini, G. Mancarella, D. Martello and A. Surdo, Astr. Part. Phys. 16 (2001) 145.

[29] C. Maieron, M.C. Martinez, J.A. Caballero and J.M. Udias, Phys. Rev. C68 (2003) 048501.

[30] A. Meucci, C. Giusti and F.D. Pacati, Nucl.Phys. A739, 277 (2004).

[31] A. Meucci, C. Giusti and F.D. Pacati, Nucl. Phys. A 744 (2004) 307

[32] B. I. S. van der Ventel and J. Piekarewicz, Phys. Rev. C 69 (2004) 035501

[33] N. Jachowicz, K. Vantournhout J. Ryckebusch and K. Heyde, Phys. Rev. Lett. 93 (2004) 082501.

[34] J. Nieves, J. E. Amaro and M. Valverde, Phys. Rev. C70, 055503 (2004) [Erratum-ibid. C72, 019902 (2005)]

[35] M. C. Martinez, P. Lava, N. Jachowicz, J. Ryckebusch, K. Vantournhout and J. M. Udias, nucl-th/0505008.

[36] K. M. Graczyk and J.T. Sobczyk, Eur. Phys. Jour. C31 (2003) 177; K. M. Graczyk, nucl-th/0401053.

[37] C. Juszczak, J.A. Nowak and J.T. Sobczyk, Eur. Phys. Jour. C39 (2005) 195.

[38] J.E. Amaro, M.B. Barbaro, J.A. Caballero, T.W. Donnelly, A. Molinari and I. Sick, Phys. Rev. C71 (2005), 015501.

[39] R. J. Glauber and G. Matthiae, Nucl. Phys. B 21 (1970) 135.

[40] J. Engel, Phys. Rev. C57 (1998) 2004.

[41] E. Oset, H. Toki and W. Weise, Phys. Rep. 83 (1982) 281.

[42] L.L. Salcedo, E. Oset, M.J. Vicente-Vacas and C. García Recio, Nucl. Phys. A484 (1988) 557.

[43] C. García-Recio, E. Oset. L.L. Salcedo, D. Strottman and M.J. Lopez, Nucl. Phys. A526 (1991) 685; J. Nieves, E. Oset, C. García-Recio, Nucl. Phys. A554 (1993) 509; ibidem Nucl. Phys. A554 (1993) 554; E. Oset, P. Fernández de Córdoba, J. Nieves, A. Ramos and L.L. Salcedo, Prog. Theor. Phys. Suppl. 117 (1994) 461; C. Albertus, J.E. Amaro and J. Nieves, Phys. Rev. Lett. 89 (2002) 032501; ibidem Phys. Rev. C67 (2003) 034604. 
[44] A. Gil, J. Nieves and E. Oset, Nucl. Phys. A627 (1997) 543.

[45] R.C. Carrasco and E. Oset, Nucl. Phys. A536 (1992) 445.

[46] M. J. Vicente Vacas and E. Oset, Nucl. Phys. A 568 (1994) 855.

[47] R.C. Carrasco, E. Oset and L.L. Salcedo, Nucl. Phys. A541 (1992) 585.

[48] R.C. Carrasco, M.J. Vicente Vacas and E. Oset, Nucl. Phys. A570 (1994) 701.

[49] A. Gil, J. Nieves and E. Oset, Nucl. Phys. A627 (1997) 599.

[50] A. Ramos, M. J. Vicente-Vacas and E. Oset, Phys. Rev. C55 (1997) 735 [Erratum-ibid. C66 (2002) 039903].

[51] Talk given by M. Valverde in 'NuFact05: 7th International Workshop on Neutrino Factories and Superbeams', Frascati, Italy, june 2005, nucl-th/0510010.

[52] J.E. Amaro, C. Maieron, J. Nieves and M. Valverde, Eur. Phys. Jour. A24 (2005) 343 [Erratum-ibid. A26, 307 (2005)].

[53] J.E. Amaro, A.M. Lallena and J. Nieves, Nucl. Phys. A623 (1997) 529.

[54] S. Galster, H. Klein, J. Moritz, K.H. Schmidt, D. Wegener and J. Bleckwenn, Nucl. Phys. B32 (1971) 221.

[55] W.M. Alberico, S.M. Bilenky, C. Giunti and C. Maieron, Z. Phys. C70 (1996) 463.

[56] G.T. Garvey, W.C. Louis and D.H. White, Phys. Rev. C48 (1993) 761.

[57] J. Speth, E. Werner and W. Wild, Phys. Rep. 33 (1977) 127; J. Speth, V. Klemt, J. Wambach and G.E. Brown Nucl. Phys. A343 (1980) 382.

[58] C. García-Recio, E. Oset and L.L. Salcedo, Phys. Rev. C37 (1988) 194.

[59] P. Fernández de Córdoba and E. Oset, Phys. Rev. C46 (1992) 1697.

[60] R.B. Firestone, Table of Isotopes ( ${ }^{\text {th }}$ Edition), John Wiley \& Sons, 1996.

[61] C.W. de Jager, H. de Vries and C. de Vries, At. Data and Nucl. Data Tables 14 (1974) 479; 36 (1987) 495.

[62] J.W. Negele and D. Vautherin, Phys. Rev. C11 (1975) 1031 and references therein.

[63] C. García-Recio, J. Nieves and E. Oset, Nucl. Phys. A547 (1992) 473 